\title{
Renormalization of Wilson Operators in the Light-Cone Gauge
}

\author{
A. Andraši \\ "Rudjer Bošković" Institute, Zagreb, Croatia
}

\begin{abstract}
We test the renormalization of Wilson operators and the Mandelstam- Leibbrandt gauge in the case when the sides of the loop are parallel to the $n, n *$ vectors used in the M-L gauge. Graphs which in the Feynman gauge are free of ultra-violet divergences, in the M-L gauge show double divergences and single divergences with non-local $\mathrm{Si}$ and $\mathrm{Ci}$ functions. These non-local functions cancel out when we add all graphs together and the constraints of gauge invariance are satisfied. In Appendix $\mathrm{C}$ we briefly discuss the problems of the M-L gauge for loops containing spacelike lines.
\end{abstract}

PACS: 11.15.Bt; 11.10.Gh

Keywords: Renormalization; Wilson operators

Electronic address:andrasi@thphys.irb.hr 


\section{Introduction.}

The aim of this research is to test the Mandelstam-Leibbrandt gauge, which is the best form of the lightcone gauge with the condition $n \cdot A=0, n^{2}=0$ (where $n$ is a vector used to define the gauge).

The Wilson operator is defined as

$$
W=\operatorname{Tr} P \exp \left(-i g \int_{C} A \cdot d x\right)
$$

where $C$ is a closed curve, $P$ denotes operator and matrix ordering along $C$, and the nonabelian gauge field $A_{\mu}$ is a matrix in some representation $R$ of the gauge group $G$. The path-ordered phase factors (1) are gauge-invariant objects and therefore an ideal laboratory for testing different gauges. Also they are better coordinates in a non-abelian theory than are the conventional vector gauge field matrices $A_{\mu}(x)$, even though they are functions of all closed paths. The gauge-variant gauge fields greatly overdescribe the observable dynamics. The operators (1) are in contrast gauge-invariant, more precisely describe the dynamics and satisfy gauge-invariant equations. The hope has therefore arisen that the $W^{\prime} s$ can replace the $A^{\prime} s$ as fundamental dynamical variables, and correspondingly that the loop functions (Wilson operators) can replace the Green's functions.

However, the loop functions are perturbatively even more divergent than the Green's functions [1]. Therefore, to make any sense out of the above program, one must renormalize. This has already been done in Lorentz gauges [2], [3]. In this paper we discuss the renormalization of Wilson operators in the Mandelstam-Leibbrandt light-cone gauge which became popular with the revival and intensive research of string theories. The complexity of the explicit calculations of individual graphs to order $g^{4}$ speaks about usefulness of the light-cone gauge in perturbative QCD for itself. Apart from that, in Appendix C, we explain why strict application of dimensional regularization in the light-cone gauge is not possible in the case of spacelike and/or timelike lines.

It was noted [1] that if the Wilson loop contains a straight light-like segment, charge renormalization does not work in a simple graph-by-graph way, but does work when certain graphs are added together. In the M-L gauge renormalization is even more complicated. We shall show to order $g^{4}$ in perturbation theory that $W$ in the M-L gauge obeys multiplicative renormalizability required in [1],

$$
W_{R}\left(A_{R} ; g_{R}\right)=Z(\epsilon) W_{B}\left(A_{B} ; g_{B}, \epsilon\right)
$$

where suffices $R$ and $B$ denote renormalized and bare quantities and dimensional regularization with $d=4-\epsilon$ is used. The relationship between $g_{B}$ and $g_{R}$ and between $A_{R}$ and $A_{B}$ should be the same as in ordinary perturbation theory. $Z(\epsilon)$ is determined from the vacuum expectation value $\langle W\rangle$

$$
\left\langle W_{R}\left(g_{R}\right)\right\rangle=Z(\epsilon)\left\langle W_{B}\left(g_{B}, \epsilon\right)\right\rangle
$$


However, the divergences of the individual graphs are not of the short distance nature and are non-local on the curve $C$. They are grouped into four tensors $n_{\beta}^{*} n_{\rho}^{*}, n_{\beta} n_{\rho}^{*}, n_{\beta}^{*} n_{\rho}$ and $n_{\beta} n_{\rho}$. There are no transverse divergences of the type e.g. $n_{\beta} P_{\rho}, P_{\beta} P_{\rho}$, (we use the decomposition of the momentum $p_{\rho}=\frac{1}{2} n_{\rho}^{*} p_{+}+\frac{1}{2} n_{\rho} p_{-}+P_{\rho}$ ), neither $g_{\beta \rho}$ divergences, as argued in Appendix A.

Let the divergent part of the amplitude for the emission of two real gluons in momentum space be

$$
M_{\beta \rho}=A n_{\beta} n_{\rho}+B n_{\beta} n_{\rho}^{*}+C n_{\beta}^{*} n_{\rho}+D n_{\beta}^{*} n_{\rho}^{*},
$$

where $M$ is the coefficient of the two fields when we expand $W$ in terms of the fields and $n$ and $n^{*}$ are the lightlike vectors used to define the gluon propagator in the M-L gauge

$$
G_{\beta \rho}=\left(k^{2}+i \eta\right)\left\{-g_{\beta \rho}+\frac{n_{\beta} k_{\rho}+n_{\rho} k_{\beta}}{n \cdot k+i \omega n^{*} \cdot k}\right\} .
$$

The polarization vectors should satisfy

$$
p \cdot e=0, q \cdot f=0
$$

and can be chosen to satisfy

$$
n \cdot e=n \cdot f=0,
$$

e.g.

$$
e_{\beta}=\frac{p_{+} n_{\beta}^{*}-p_{-} n_{\beta}-2 p_{\beta}}{\left|4 p_{+} p_{-}\right|^{1 / 2}}, f_{\rho}=\frac{q_{+} n_{\rho}^{*}-q_{-} n_{\rho}-2 q_{\rho}}{\left|4 q_{+} q_{-}\right|^{1 / 2}}
$$

for $p$ and $q$ on shell respectively. The other independent polarization vector which is perpendicular to $P, n$ and $n^{*}$ and counterpart to $e$, gives zero when contracted into $M_{\beta \rho}$ and so plays no role in this paper. Of course, there is a counterpart of $f$ as well.

We have four identities following from gauge invariance, which the amplitude should satisfy.

(a) $M^{\beta \rho} e_{\beta} f_{\rho}$ should be the same as Feynman gauge when the external momenta $p$ and $q$ are on shell,

(b) $M^{\beta \rho} e_{\beta} q_{\rho}=0$ for $p$ on shell,

$M^{\beta \rho} p_{\beta} f_{\rho}=0$ for $q$ on shell,

(c) $M^{\beta \rho} p_{\beta} q_{\rho}=0$.

These equations allow us to redefine the vectors (5) into

$$
e_{\beta}^{\prime}=p_{-} n_{\beta}-p_{+} n_{\beta}^{*}, f_{\rho}^{\prime}=q_{-} n_{\rho}-q_{+} n_{\rho}^{*},
$$

and the tensor structure (4) which satisfies (b) and (c) can be written in the form

$$
M_{\beta \rho}=e_{\beta}^{\prime} f_{\rho}^{\prime} M .
$$


The form (7) will in section 5 be crucial to show that non-local divergences must cancel. $(a),(b)$ and $(c)$ impose the constraint on (4)

$$
\begin{aligned}
& p_{+} q_{+} A+p_{+} q_{-} B-p_{-} q_{+} C-p_{-} q_{-} D=0, \\
& p_{+} q_{+} A-p_{+} q_{-} B+q_{+} p_{-} C-p_{-} q_{-} D=0 \\
& p_{+} q_{+} A+p_{+} q_{-} B+p_{-} q_{+} C+p_{-} q_{-} D=0,
\end{aligned}
$$

i.e.

$$
q_{+} A+q_{-} B=0,
$$

and fix all the ratios of $A: B: C: D$. The answers we find in eqs. (15), (22) and (32) confirm this prediction and are invariant under $n->c n, n^{*}>c n^{*}, p_{+}->c p_{+}$, $p_{-}->c p_{-}$for any constant $c$.

$A, B, C$ and $D$ turn out to be local, although from the example of the self-energy graph [4], one might have expected non-local divergences to occurr with the Wilson loop. If we take a self-energy part and try to derive an on-shell physical thing, we get zero (we take $S_{\beta \rho}(p)$, put $p^{2}=0$, multiply by $e_{\beta} e_{\rho}$ where $e$ is a polarization vector satisfying $p \cdot e=0)$. Therefore we cannot deduce much by arguing that physical things are gauge invariant - we get just $0=0$. But, for the Wilson loop, we do not get zero if we put $p^{2}=q^{2}=0$ and multiply by polarization vectors. So the gauge-independence argument does give some information. As the Feynman gauge non-local divergences cancel [1], $(a)$, $(b)$ and $(c)$ explain why there are no non-local divergences in the M-L Wilson operator.

The abelian $C_{R} C_{R}$ part obeys the factorization theorem [5], [6]. Therefore in this work we shall concentrate only on the non-abelian $C_{G} C_{R}$ part of the graphs, where $C_{R}$ and $C_{G}$ are the Casimirs for the representation used to define the Wilson loop and the gluons. In the following sections we list final results for the amplitude $M_{\beta \rho}$ to order $g^{4}$ after the decomposition in (4). The graphs are grouped into sets according to their topological equivalence. 


\section{The $n_{\beta}^{*} n_{\rho}^{*}$ sector of $W_{B}$}

We list the final results for groups of graphs shown in the figures. The multiplication by the overall factor $C_{\beta \rho}$ is understood for each graph.

$$
\begin{gathered}
M_{\beta \rho}=C_{\beta \rho} M \\
C_{\beta \rho}=\frac{2}{\epsilon} g^{4} C_{G} \operatorname{Tr}\left(t_{b} t_{d}\right) n_{\beta}^{*} n_{\rho}^{*} \pi^{2-\frac{\epsilon}{2}}(2 \pi)^{-n}
\end{gathered}
$$

We denote the frequent non-local functions which appear in all equations by

$$
\begin{gathered}
\operatorname{Ci}(x)=\int_{0}^{x} \frac{\cos t-1}{t} d t \\
\operatorname{Si}(x)=\int_{0}^{x} \frac{\sin t}{t} d t
\end{gathered}
$$

\section{A-set}

The graphs contributing to the A-set are shown in Fig.1. There are also graphs with $p$ and $q$ interchanged. Then the ultra-violet divergent part of the graphs in Fig.1 is

$$
\begin{gathered}
\left(M_{1}+M_{2}\right)(A)=-\frac{1}{p_{-} q_{-}}\left(e^{-i p_{+} T}+e^{-i q_{+} T}\right)\left(e^{-i q_{-} L}-1\right) \times\left\{2\left(e^{-i p_{-} L}-1\right)\right. \\
\left.+\left(e^{-i p_{-} L}-1\right) \mathrm{Ci}\left(p_{-} L\right)+i\left(e^{-i p_{-} L}+1\right) \operatorname{Si}\left(p_{-} L\right)\right\}
\end{gathered}
$$

B - set

$B$-graphs are shown in Fig.2. Addition of symmetric graphs is understood.

$$
\begin{gathered}
\left(M_{1}+M_{2}+\ldots+M_{6}\right)(B)=\left(e^{-i T r+}+1\right) \times\left\{\frac{2}{p_{-} q_{-}}\left(e^{-i p_{-} L}-1\right)\left(e^{-i q_{-} L}-1\right)\right. \\
+\left(\frac{2}{p_{-} q_{-}}+\frac{2}{p_{-} r_{-}}\right)\left[\left(e^{-i r_{-} L}+1\right) \operatorname{Ci}\left(r_{-} L\right)+i\left(e^{-i r_{-} L}-1\right) \operatorname{Si}\left(r_{-} L\right)\right] \\
-\left(\frac{2}{p_{-} q_{-}}+\frac{2}{p_{-} r_{-}}\right)\left[\left(e^{-i r_{-} L}+1\right) \mathrm{Ci}\left(q_{-} L\right)+i\left(e^{-i r_{-} L}-1\right) \operatorname{Si}\left(q_{-} L\right)\right] \\
\quad-\frac{2}{p_{-} r_{-}}\left[\left(e^{-i r_{-} L}+1\right) \operatorname{Ci}\left(p_{-} L\right)+i\left(e^{-i r_{-} L}-1\right) \operatorname{Si}\left(p_{-} L\right)\right] \\
\left.-\frac{1}{p_{-} q_{-}}\left(e^{-i q_{-} L}+1\right)\left[\left(e^{-i p_{-} L}+1\right) \operatorname{Ci}\left(p_{-} L\right)+i\left(e^{-i p_{-} L}-1\right) \operatorname{Si}\left(p_{-} L\right)\right]\right\}
\end{gathered}
$$


where

$$
r=p+q
$$

C - set

The $C$ graphs are shown in Fig.3.

$$
\begin{gathered}
\left(M_{1}+M_{2}+M_{3}+M_{4}\right)(C)=\frac{2}{q_{-} r_{-}}\left(e^{-i T p_{+}}+e^{-i T q_{+}}\right) \\
\times\left\{\left(e^{-i r_{-} L}+1\right)\left[\mathrm{Ci}\left(q_{-} L\right)-\operatorname{Ci}\left(p_{-} L\right)\right]+i\left(e^{-i r_{-} L}-1\right)\left[\operatorname{Si}\left(q_{-} L\right)-\operatorname{Si}\left(p_{-} L\right)\right]\right\} \\
+\frac{2}{q_{-} r_{-}}\left(e^{-i T r_{+}}+1\right)\left[\left(e^{-i r_{-} L}+1\right) \operatorname{Ci}\left(r_{-} L\right)+i\left(e^{-i r_{-} L}-1\right) \operatorname{Si}\left(r_{-} L\right)\right] \\
-\frac{1}{q_{-} p_{-}}\left(e^{-i T p_{+}}-1\right)\left(e^{-i T q_{+}}-1\right)\left(e^{-i L q_{-}}+1\right)\left[\left(e^{-i p_{-} L}+1\right) \operatorname{Ci}\left(p_{-} L\right)+i\left(e^{-i p_{-} L}-1\right) \operatorname{Si}\left(p_{-} L\right)\right] \\
+\frac{i \pi}{p_{-} q_{-}}\left(e^{-i T p_{+}}+1\right)\left(e^{-i T q_{+}}-1\right)\left(e^{-i p_{-} L}-1\right)\left(e^{-i q_{-} L}+1\right) \\
-\frac{2 i \pi}{q_{-} r_{-}}\left(e^{-i T p_{+}}+1\right)\left(e^{-i T q_{+}}-1\right)\left(e^{-i r_{-} L}-1\right)
\end{gathered}
$$

Again we have to add the symmetric graphs with $p$ and $q$ interchanged.

D - set

The complete set of $D$ graphs (including symmetric graphs) is shown in Fig.4.

$$
\begin{gathered}
\left(M_{1}+M_{2}+\ldots+M_{8}\right)(D)=-\frac{2}{p_{-} q_{-}}\left(e^{-i T r_{+}}+1\right) \\
\times\left\{2\left(e^{-i r_{-} L}+1\right) \operatorname{Ci}\left(r_{-} L\right)+2 i\left(e^{-i r_{-} L}-1\right) \operatorname{Si}\left(r_{-} L\right)\right. \\
-\left(e^{-i p_{-} L}+1\right)\left[\left(e^{-i q_{-} L}+1\right) \operatorname{Ci}\left(q_{-} L\right)+i\left(e^{-i q_{-} L}-1\right) \operatorname{Si}\left(q_{-} L\right)\right] \\
\left.-\left(e^{-i q_{-} L}+1\right)\left[\left(e^{-i p_{-} L}+1\right) \operatorname{Ci}\left(p_{-} L\right)+i\left(e^{-i p_{-} L}-1\right) \operatorname{Si}\left(p_{-} L\right)\right]\right\} \\
-2\left(\frac{1}{q_{-} r_{-}}-\frac{1}{p_{-} r_{-}}\right)\left(e^{-i T p_{+}}+e^{-i T q_{+}}\right) \\
\times\left\{\left(e^{-i r_{-} L}+1\right)\left[\mathrm{Ci}\left(q_{-} L\right)-\mathrm{Ci}\left(p_{-} L\right)\right]+i\left(e^{-i r_{-} L}-1\right)\left[\operatorname{Si}\left(q_{-} L\right)-\operatorname{Si}\left(p_{-} L\right)\right]\right\} \\
-\frac{2}{p_{-} q_{-}}\left(e^{-i T p_{+}}+e^{-i T q_{+}}\right)\left[\left(e^{-i q_{-} L}+1\right) \operatorname{Ci}\left(q_{-} L\right)+i\left(e^{-i q_{-} L}-1\right) \operatorname{Si}\left(q_{-} L\right)\right] \\
-\frac{2}{p_{-} q_{-}}\left(e^{-i T p_{+}}+e^{-i T q_{+}}\right)\left[\left(e^{-i p_{-} L}+1\right) \operatorname{Ci}\left(p_{-} L\right)+i\left(e^{-i p_{-} L}-1\right) \operatorname{Si}\left(p_{-} L\right)\right] \\
-\frac{2}{p_{-} q_{-}}\left(e^{-i T p_{+}}+e^{-i T q_{+}}\right)\left(e^{-i p_{-} L}-1\right)\left[\operatorname{Ci}\left(q_{-} L\right)-i \operatorname{Si}\left(q_{-} L\right)\right]
\end{gathered}
$$




$$
\begin{gathered}
-\frac{2}{p_{-} q_{-}}\left(e^{-i T p_{+}}+e^{-i T q_{+}}\right)\left(e^{-i q_{-} L}-1\right)\left[\operatorname{Ci}\left(p_{-} L\right)-i \operatorname{Si}\left(p_{-} L\right)\right] \\
-\frac{2 i \pi}{p_{-} q_{-}}\left(e^{-i T p_{+}}-e^{-i T q_{+}}\right)\left(e^{-i q_{-} L}-e^{-i p_{-} L}\right) \\
-2 i \pi\left(\frac{1}{p_{-} r_{-}}-\frac{1}{q_{-} r_{-}}\right)\left(e^{-i r_{-} L}-1\right)\left(e^{-i T q_{+}}-e^{-i T p_{+}}\right)
\end{gathered}
$$

E - set

E-set is shown in Fig.5.

$$
\begin{aligned}
\left(M_{1}+\ldots+M_{4}\right)(E) & =-4\left(e^{-i T r_{+}}+1\right) \times\left\{\frac{1}{p_{-} q_{-}}\left[\left(e^{-i r_{-} L}+1\right) \operatorname{Ci}\left(r_{-} L\right)+i\left(e^{-i r_{-} L}-1\right) \operatorname{Si}\left(r_{-} L\right)\right]\right. \\
& -\frac{1}{q_{-} r_{-}}\left[\left(e^{-i r_{-} L}+1\right) \operatorname{Ci}\left(p_{-} L\right)+i\left(e^{-i r_{-} L}-1\right) \operatorname{Si}\left(p_{-} L\right)\right] \\
& \left.-\frac{1}{p_{-} r_{-}}\left[\left(e^{-i r_{-} L}+1\right) \operatorname{Ci}\left(q_{-} L\right)+i\left(e^{-i r_{-} L}-1\right) \operatorname{Si}\left(q_{-} L\right)\right]\right\}
\end{aligned}
$$

The complete sum of all the graphs contributing to the $n^{*} n^{*}$ sector is very simple.

$$
\begin{gathered}
S_{\beta \rho}\left(n^{*} n^{*}\right)=\frac{8}{\epsilon} g^{4} C_{G} \operatorname{Tr}\left(t_{b} t_{d}\right) n_{\beta}^{*} n_{\rho}^{*} \pi^{2-\frac{\epsilon}{2}}(2 \pi)^{-4} \\
\times \frac{1}{p_{-} q_{-}}\left(e^{-i T q_{+}}-1\right)\left(e^{-i T p_{+}}-1\right)\left(e^{-i q_{-} L}-1\right)\left(e^{-i p_{-} L}-1\right) \\
=\frac{8}{\epsilon} g^{2} C_{G} \pi^{2}(2 \pi)^{-4} B_{\beta \rho}\left(n^{*} n^{*}\right)
\end{gathered}
$$

where $B_{\beta \rho}\left(n^{*} n^{*}\right)$ denotes the Born term which is in the $n^{*} n^{*}$ sector only. The non-local functions have cancelled out. This result alone does not prove renormalizability as the field renormalization matrix in the lightcone gauge mixes all three sectors. 


\section{The $n_{\beta} n_{\rho}$ sector of $W_{B}$}

Again we list the final results, but here the overall factor is

$$
\begin{gathered}
C_{\beta \rho}^{\prime}=\frac{2}{\epsilon} g^{4} C_{G} \operatorname{Tr}\left(t_{b} t_{d}\right) n_{\beta} n_{\rho} \pi^{2-\frac{\epsilon}{2}}(2 \pi)^{-n} \frac{1}{q_{+} p_{+}}, \\
M_{\beta \rho}=C_{\beta \rho}^{\prime} M
\end{gathered}
$$

G2 - set

The sum of the three graphs of the G2-set shown in Fig.(6) is

$$
\begin{gathered}
M(G 2)=-\frac{8}{\epsilon}\left(e^{-i r_{-} L}+1\right)\left(e^{-i T p_{+}}-1\right)\left(e^{-i T q_{+}}-1\right) \\
-2\left(e^{-i T p_{+}}-1\right)\left(e^{-i T q_{+}}-1\right)\left\{\left(e^{-i r_{-} L}+1\right)\left[\operatorname{Ci}\left(r_{-} L\right)+2 \ln \left(T L \mu^{2}\right)+i \pi\right]\right. \\
\left.+i\left(e^{-i r_{-} L}-1\right) \operatorname{Si}\left(r_{-} L\right)\right\} \\
-2\left(e^{-i T q_{+}}+e^{-i T p_{+}}\right)\left\{\left(e^{-i r_{-} L}+1\right)\left[\operatorname{Ci}\left(r_{-} L\right)-\operatorname{Ci}\left(q_{-} L\right)-\operatorname{Ci}\left(p_{-} L\right)\right]\right. \\
\left.+i\left(e^{-i r_{-} L}-1\right)\left[\operatorname{Si}\left(r_{-} L\right)-\operatorname{Si}\left(q_{-} L\right)-\operatorname{Si}\left(p_{-} L\right)\right]\right\}
\end{gathered}
$$

G1 - set

G1-set of graphs are the graphs with one 3-gluon vertex. There are two groups of such graphs. The two graphs shown in Fig.7 give

$$
\begin{gathered}
M^{a}(G 1)=\left(e^{-i T p_{+}}-1\right)\left(e^{-i T q_{+}}-1\right)\left(e^{-i q_{-} L}+1\right) \\
\times\left\{\left(e^{-i p_{-} L}+1\right)\left[\mathrm{Ci}\left(p_{-} L\right)+2 \gamma+\frac{4}{\epsilon}+i \pi+2 \ln \left(T L \mu^{2}\right)\right]+i\left(e^{-i p_{-} L}-1\right) \operatorname{Si}\left(p_{-} L\right)\right\} .
\end{gathered}
$$

Of course the graphs with $p$ and $q$ interchanged must be added. The graphs in Fig. 8 give

$$
\begin{gathered}
M^{b}(G 1)=\left(e^{-i T p_{+}}-1\right)\left(e^{-i T q_{+}}-1\right)\left(e^{-i q_{-} L}-1\right) \\
\times\left\{2\left(e^{-i p_{-} L}-1\right)+\left(e^{-i p_{-} L}-1\right) \operatorname{Ci}\left(p_{-} L\right)+i\left(e^{-i p_{-} L}+1\right) \operatorname{Si}\left(p_{-} L\right)\right\} .
\end{gathered}
$$

Again there is a symmetric set of graphs with $p$ and $q$ interchanged.

\section{$\mathbf{G}(\mathbf{L}+\mathbf{R})$ - set}

Adding the symmetric graphs to Fig.9, the total sum of 4 graphs is

$$
\begin{aligned}
G(L+R)= & -2\left(e^{-i T r_{+}}+1\right)\left\{\left(e^{-i r_{-} L}+1\right)\left[\mathrm{Ci}\left(p_{-} L\right)+\mathrm{Ci}\left(q_{-} L\right)-\mathrm{Ci}\left(r_{-} L\right)\right]\right. \\
& \left.+i\left(e^{-i r_{-} L}-1\right)\left[\operatorname{Si}\left(p_{-} L\right)+\operatorname{Si}\left(q_{-} L\right)-\operatorname{Si}\left(r_{-} L\right)\right]\right\} .
\end{aligned}
$$


G0 - set

Fig.10 gives

$$
\begin{gathered}
M(G 0)=-\frac{8}{\epsilon}\left(e^{-i T p_{+}}-1\right)\left(e^{-i T q_{+}}-1\right)\left(e^{-i q_{-} L}+e^{-i p_{-} L}\right) \\
\times\left(\mu^{2} T L\right)^{\frac{\epsilon}{2}}\left(1+\gamma \frac{\epsilon}{2}+\frac{i \pi \epsilon}{4}\right) .
\end{gathered}
$$

The sum of all the graphs contributing to the $n n$ sector is

$$
\begin{gathered}
S_{\beta \rho}(n n)=\frac{8}{\epsilon}\left(e^{-i T p_{+}}-1\right)\left(e^{-i T q_{+}}-1\right)\left(e^{-i p_{-} L}-1\right)\left(e^{-i q_{-} L}-1\right) \frac{1}{q_{+} p_{+}} \\
\times g^{4} C_{G} \operatorname{Tr}\left(t_{b} t_{d}\right) n_{\beta} n_{\rho} \pi^{2-\frac{\epsilon}{2}}(2 \pi)^{-4} \\
=\frac{8}{\epsilon} g^{2} C_{G} \pi^{2}(2 \pi)^{-4} B_{\beta \rho}(n n)
\end{gathered}
$$

where $B_{\beta \rho}(n n)$ is the $g^{2}$ term for the $n n$ sector of $W_{B}\left(g_{B}, \epsilon\right)$.

4. The $n_{\beta} n_{\rho}^{*}$ sector of $W_{B}$

The final results for the $n_{\rho} n_{\beta}^{*}$ sector we get from $n_{\beta} n_{\rho}^{*}$ by the change $p, b, \beta$ into $q, d, \rho$. The overall factor for all the graphs in this sector is

$$
\begin{gathered}
C_{\beta \rho} "=\frac{2}{\epsilon} g^{4} C_{G} \operatorname{Tr}\left(t_{b} t_{d}\right) n_{\beta} n_{\rho}^{*} \pi^{2-\frac{\epsilon}{2}}(2 \pi)^{-n}, \\
M_{\beta \rho}=C_{\beta \rho} " M .
\end{gathered}
$$

A - set

The graphs in Fig.11 give

$$
\begin{gathered}
M(A)=\left(e^{-i T q_{+}}+e^{-i T p_{+}}\right)\left(e^{-i q_{-} L}-1\right) \frac{1}{p_{+} q_{-}} \\
\times\left\{2\left(e^{-i p_{-} L}-1\right)+\left(e^{-i p_{-} L}-1\right) \operatorname{Ci}\left(p_{-} L\right)+i\left(e^{-i p_{-} L}+1\right) \operatorname{Si}\left(p_{-} L\right)\right\} .
\end{gathered}
$$

$A^{\prime}$ - set

The $A^{\prime}$-set is presented in Fig. 12 .

$$
\begin{gathered}
M\left(A^{\prime}\right)=-\left(e^{-i T p_{+}}-1\right)\left(e^{-i T q_{+}}-1\right)\left(e^{-i p_{-} L}-1\right) \frac{1}{p_{+} q_{-}} \\
\times\left\{2\left(e^{-i q_{-} L}-1\right)+\left(e^{-i q_{-} L}-1\right) \operatorname{Ci}\left(q_{-} L\right)+i\left(e^{-i q_{-} L}+1\right) \operatorname{Si}\left(q_{-} L\right)\right.
\end{gathered}
$$


B - set

$B$ - set is shown in Fig.13.

$$
\begin{gathered}
M(B)=-\left(e^{-i T r_{+}}+1\right) \times\left\{\frac{2}{p_{+} q_{-}}\left(e^{-i p_{-} L}-1\right)\left(e^{-i q_{-} L}-1\right)\right. \\
+\left(\frac{2}{p_{+} q_{-}}+\frac{2}{p_{+} r_{-}}\right)\left[\left(e^{-i r_{-} L}+1\right) \mathrm{Ci}\left(r_{-} L\right)+i\left(e^{-i r_{-} L}-1\right) \operatorname{Si}\left(r_{-} L\right)\right] \\
-\left(\frac{2}{p_{+} q_{-}}+\frac{2}{p_{+} r_{-}}\right)\left[\left(e^{-i r_{-} L}+1\right) \mathrm{Ci}\left(q_{-} L\right)+i\left(e^{-i r_{-} L}-1\right) \operatorname{Si}\left(q_{-} L\right)\right] \\
\quad+\frac{2}{p_{+} r_{-}}\left[\left(e^{-i r_{-} L}+1\right) \operatorname{Ci}\left(p_{-} L\right)+i\left(e^{-i r_{-} L}-1\right) \operatorname{Si}\left(p_{-} L\right)\right] \\
\left.-\frac{1}{p_{+} q_{-}}\left(e^{-i q_{-} L}+1\right)\left[\left(e^{-i p_{-} L}+1\right) \operatorname{Ci}\left(p_{-} L\right)+i\left(e^{-i p_{-} L}-1\right) \operatorname{Si}\left(p_{-} L\right)\right]\right\}
\end{gathered}
$$

\section{C - set}

Graphs grouped into the $C$-set are shown in Fig.14.

$$
\begin{gathered}
M(C)=\frac{2}{p_{+} q_{-}}\left[\frac{2}{\epsilon}+\ln \left(T L \mu^{2}\right)+\gamma\right]\left(e^{-i T p_{+}}-1\right)\left(e^{-i T q_{+}}-1\right)\left(e^{-i p_{-} L}-1\right)\left(e^{-i q_{-} L}-1\right) \\
+2\left(e^{-i T r_{+}}+1\right)\left(\frac{1}{q_{-} p_{+}}+\frac{1}{r_{-} p_{+}}\right)\left\{\left(e^{-i r_{-} L}+1\right) \mathrm{Ci}\left(r_{-} L\right)+i\left(e^{-i r_{-} L}-1\right) \operatorname{Si}\left(r_{-} L\right)\right\} \\
-2\left(e^{-i T p_{+}}+e^{-i T q_{+}}\right)\left(\frac{1}{q_{-} p_{+}}+\frac{1}{r_{-} p_{+}}\right)\left\{\left(e^{-i r_{-} L}+1\right) \mathrm{Ci}\left(q_{-} L\right)+i\left(e^{-i r_{-} L}-1\right) \operatorname{Si}\left(q_{-} L\right)\right\} \\
+\left\{\frac{2}{r_{-} p_{+}}\left(e^{-i T p_{+}}+e^{-i T q_{+}}\right)-\frac{1}{q_{-} p_{+}}\left(e^{-i T r_{+}}+1\right)\left(e^{-i q_{-} L}+1\right)\right. \\
\left.\quad+\frac{1}{q_{-} p_{+}}\left(e^{-i T p_{+}}+e^{-i T q_{+}}\right)\left(e^{-i q_{-} L}-1\right)\right\} \\
\quad \times\left\{\mathrm{Ci}\left(p_{-} L\right)-i \operatorname{Si}\left(p_{-} L\right)\right\} \\
\frac{2}{r_{-} p_{+}} e^{-i r_{-} L}\left(e^{-i T p_{+}}+e^{-i T q_{+}}\right)-\frac{1}{q_{-} p_{+}} e^{-i p_{-} L}\left(e^{-i q_{-} L}+1\right)\left(e^{-i T r_{+}}+1\right) \\
\quad \frac{1}{q_{-} p_{+}}\left(e^{-i T p_{+}}-1\right)\left(e^{-i T q_{+}}-1\right)\left(e^{-i p_{-} L}-1\right)\left(e^{-i q_{-} L}-1\right) \\
\quad \times\left\{\mathrm{Ci}\left(p_{-} L\right)+i \operatorname{Si}\left(p_{-} L\right)\right\} \\
\left.\left.q_{-}^{-i q_{-} L}-1\right)\left(e^{-i T p_{+}}+e^{-i T q_{+}}\right)\right\}
\end{gathered}
$$




$$
-\frac{2 i \pi}{r_{-} p_{+}}\left(e^{-i T p_{+}}-1\right)\left(e^{-i T q_{+}}+1\right)\left(e^{-i r_{-} L}-1\right) .
$$

C'- set

The two graphs of $C^{\prime}$-set in Fig.15 give

$$
\begin{gathered}
M\left(C^{\prime}\right)=\frac{1}{q_{-} p_{+}}\left(e^{-i T p_{+}}-1\right)\left(e^{-i p_{-} L}+1\right) \\
\times\left\{\left(e^{-i T q_{+}}-1\right)\left[\left(e^{-i q_{-} L}+1\right) \operatorname{Ci}\left(q_{-} L\right)+i\left(e^{-i q_{-} L}-1\right) \operatorname{Si}\left(q_{-} L\right)\right]-3 i \pi\left(e^{-i q_{-} L}-1\right)\right\}
\end{gathered}
$$

D - set

$D$-set is shown in Fig.16.

$$
\begin{aligned}
& M(D)=-\frac{2}{q_{-} p_{+}}\left[\frac{2}{\epsilon}+\ln \left(T L \mu^{2}\right)+\frac{i \pi}{2}+\gamma\right] \times\left(e^{-i T p_{+}}-1\right)\left(e^{-i T q_{+}}-1\right)\left(e^{-i p_{-} L}-1\right)\left(e^{-i q_{-} L}-1\right) \\
& -\frac{2}{q_{-} p_{+}}\left(e^{-i T p_{+}}-1\right)\left(e^{-i T q_{+}}-1\right)\left(e^{-i p_{-} L}+1\right) \\
& \times\left\{\left(e^{-i q_{-} L}+1\right) \mathrm{Ci}\left(q_{-} L\right)+i\left(e^{-i q_{-} L}-1\right)\left(\operatorname{Si}\left(q_{-} L\right)-\frac{\pi}{2}\right)\right\} \\
& +\frac{2 i \pi}{q_{-} p_{+}}\left(e^{-i T p_{+}}-1\right)\left(e^{-i p_{-} L}+1\right)\left(e^{-i q_{-} L}-1\right)
\end{aligned}
$$

$E$-set contains graphs with two 3 -gluon vertices and the graph with the 4-gluon vertex spanning across the loop. They are shown in Fig.17.

$$
\begin{aligned}
& \quad M(E)=-\frac{2}{p_{+} r_{-}} \times\left\{\left(e^{-i T r_{+}}+1\right)\left[\left(e^{-i r_{-} L}+1\right) \operatorname{Ci}\left(r_{-} L\right)+i\left(e^{-i r_{-} L}-1\right) \operatorname{Si}\left(r_{-} L\right)\right]\right. \\
& +\left(e^{-i T p_{+}}+e^{-i T q_{+}}\right)\left[\left(e^{-i r_{-} L}+1\right)\left(\operatorname{Ci}\left(p_{-} L\right)-\operatorname{Ci}\left(q_{-} L\right)\right)+i\left(e^{-i r_{-} L}-1\right)\left(\operatorname{Si}\left(p_{-} L\right)-\operatorname{Si}\left(q_{-} L\right)\right)\right] \\
& \left.\quad-i \pi\left(e^{-i r_{-} L}-1\right)\left(e^{-i T q_{+}}+1\right)\left(e^{-i T p_{+}}-1\right)\right\} \\
& \text { F - set }
\end{aligned}
$$

The four graphs of the $F$-set are shown in Fig.18. Of course, there are also symmetric graphs with $p$ and $q$ interchanged. The complete sum of eight graphs amounts to

$$
M(F)=\frac{2}{p_{+} r_{-}}\left(e^{-i T r_{+}}+1\right) \times\left\{\left(e^{-i r_{-} L}+1\right)\left[\mathrm{Ci}\left(p_{-} L\right)-\mathrm{Ci}\left(q_{-} L\right)+\mathrm{Ci}\left(r_{-} L\right)\right]\right.
$$




$$
\left.+i\left(e^{-i r_{-} L}-1\right)\left[\operatorname{Si}\left(p_{-} L\right)-\operatorname{Si}\left(q_{-} L\right)+\operatorname{Si}\left(r_{-} L\right)\right]\right\} .
$$

The total sum for the $n n^{*}$ sector is

$$
\begin{gathered}
S_{\beta \rho}\left(n n^{*}\right)=-\frac{8}{\epsilon} g^{4} C_{G} \operatorname{Tr}\left(t_{b} t_{d}\right) n_{\beta} n_{\rho}^{*} \pi^{2-\frac{\epsilon}{2}}(2 \pi)^{-n} \\
\times \frac{1}{p_{+} q_{-}}\left(e^{-i T p_{+}}-1\right)\left(e^{-i T q_{+}}-1\right)\left(e^{-i p_{-} L}-1\right)\left(e^{-i q_{-} L}-1\right) \\
-\frac{8}{\epsilon} g^{4} C_{G} \operatorname{Tr}\left(t_{b} t_{d}\right) n_{\rho} n_{\beta}^{*} \pi^{2-\frac{\epsilon}{2}}(2 \pi)^{-n} \\
\times \frac{1}{q_{+} p_{-}}\left(e^{-i T p_{+}}-1\right)\left(e^{-i T q_{+}}-1\right)\left(e^{-i p_{-} L}-1\right)\left(e^{-i q_{-} L}-1\right)
\end{gathered}
$$

This is again proportional to the $g^{2}$ term with the same factor $\frac{8}{\epsilon}$ as in eq.(15) and eq.(22).

\section{Discussion}

We are now going to explain how (2) works out to order $g^{4}$. The field renormalization matrix in the M-L gauge is [8],[9],[10], in momentum space, where $A(p)$ is the gluon field in momentum space

$$
\begin{aligned}
A_{\beta}^{B}(p)=\left(1+\frac{11}{6} c\right)\left[g_{\beta \gamma}\right. & \left.-c n_{\beta}\left(n_{\gamma}^{*}-\frac{n^{*} \cdot p}{n \cdot p+i \eta n^{*} \cdot p} n_{\gamma}\right)\right] A^{\gamma R}(p) \\
& =z_{\beta \gamma} A^{\gamma R}(p)
\end{aligned}
$$

where

$$
c=\frac{g_{R}^{2}}{8 \pi^{2} \epsilon} C_{G}
$$

and coupling constant renormalization is

$$
g_{B}=\left(1-\frac{11}{6} c\right) g_{R}
$$

On the right hand side (2) contains various sorts of fourth order terms.

(a) $W_{B}$ to fourth order, $Z, z$ and $g_{B}$ to zeroth order

(b) $W_{B}$ to second order, $Z$ to second order

(c) $W_{B}$ to second, $g_{B}$ to second

(d) $W_{B}$ to second, $z$ to second

Then (b) contributes only to the abelian $C_{R} C_{R}$ part, while (c) and (d) should give the counter-terms needed to cancel the UV divergences we found in (a). Of course, since $W_{B}$ to second order has two real gluons, that is two $A_{B}$ operators, it gets two $z$ factors, one depending on $p$ and the other on $q$. We list counter-terms for each sector separately. 
(1) $n^{*} n^{*}$ sector

Although the Born term is contained in the $n^{*} n^{*}$ sector only, we had to study the off-shell sectors as well. The reason is the field renormalization matrix $z_{\beta \gamma}$ which mixes all three sectors. The Born term to order $g^{2}$ is

$$
B_{\beta \rho}\left(n^{*} n^{*}\right)=\frac{1}{p_{-} q_{-}} g_{B}^{2} n_{\beta}^{*} n_{\rho}^{*} H A_{B}^{\beta}(p) A_{B}^{\rho}(q)
$$

where

$$
H=\operatorname{Tr}\left(t_{b} t_{d}\right)\left(e^{-i T p_{+}}-1\right)\left(e^{-i T q_{+}}-1\right)\left(e^{-i L p_{-}}-1\right)\left(e^{-i L q_{-}}-1\right) .
$$

To order $g_{R}^{4}$ the operator $(z-1) W_{B}+\left(g_{B}-g_{R}\right) W_{B}$ on the right of eq.(2) gives for the $n^{*} n^{*}$ sector the counter-term

$$
\begin{gathered}
W_{\beta \rho}^{c t}\left(n^{*} n^{*}\right)=n_{\beta}^{*} n_{\rho}^{*} \frac{g_{R}^{2}}{p_{-} q_{-}} H\left[\frac{11}{6} c g^{\beta \gamma}-c n^{\beta}\left(n^{\gamma *}-\frac{n^{*} \cdot p}{n \cdot p} n^{\gamma}\right)\right] A_{\gamma}^{R}(p) A_{\rho}^{R}(q) \\
+n_{\beta}^{*} n_{\rho}^{*} \frac{g_{R}^{2}}{p_{-} q_{-}} H\left[\frac{11}{6} c g^{\rho \gamma}-c n^{\rho}\left(n^{\gamma *}-\frac{n^{*} \cdot q}{n \cdot q} n^{\gamma}\right)\right] A_{\gamma}^{R}(q) A_{\beta}^{R}(p) \\
-2 n_{\beta}^{*} n_{\rho}^{*} \frac{11}{6} c H \frac{g_{R}^{2}}{p_{-} q_{-}} A^{\beta R}(p) A^{\rho R}(q) .
\end{gathered}
$$

We notice that the factor $\frac{11}{6} c$ cancels out between the wave function renormalization (two first terms) and the coupling constant renormalization (last term). Hence, the counter-term to order $g_{R}^{4}$ for the $n^{*} n^{*}$ sector is

$$
W_{\beta \rho}^{c t}\left(n^{*} n^{*}\right)=-4 c n_{\rho}^{*} n_{\beta}^{*} \frac{1}{p_{-} q_{-}} H+2 c\left[n_{\beta}^{*} n \rho \frac{1}{p_{-} q_{+}}+n_{\rho}^{*} n_{\beta} \frac{1}{q_{-} p_{+}}\right] H .
$$

(2) $n n^{*}$ sector

$$
W_{\beta \rho}^{c t}\left(n n^{*}\right)=-4 c n_{\rho} n_{\beta} \frac{1}{p_{+} q_{+}} H+2 c\left[n_{\beta}^{*} n_{\rho} \frac{1}{p_{-} q_{+}}+n_{\rho}^{*} n_{\beta} \frac{1}{p_{+} q_{-}}\right] H
$$

(3) $n n$ sector gives zero

The sum of (1) and (2) gives exactly the counter-terms needed to cancel (15), (22) and (32). The complications with non-local $\mathrm{Si}$ and $\mathrm{Ci}$ divergences were caused by the choice of the M-L gauge, not the lightlike sides of the Wilson loop, as shown in Appendix C. We certainly expected (a) of eq.(6) to be gauge-invariant, but in fact we find that the whole of the divergent part of $M_{\beta \rho}$ is gauge invariant.

We shall now explain how the field renormalization matrix $z_{\beta \gamma}$ leaves this tensor structure unchanged. Let us envoke the tensor structure for the amplitude in eq.(7)

$$
M_{\beta \rho}=e_{\beta}^{\prime} f_{\rho}^{\prime} M .
$$


This vanishes when contracted with $p_{\beta}$ or $q_{\rho}$. Then it is easy to see why $z_{\beta \gamma}$ does not change the structure. The non-local structure in $z_{\beta \gamma}$ contains

$$
n_{\beta}\left(n_{\gamma}^{*}-\frac{n^{*} \cdot p}{n \cdot p} n_{\gamma}\right)=-\frac{n_{\beta} e_{\gamma}^{\prime}}{p_{+}}
$$

When contracted with $M_{\beta \rho}$ the term $\frac{n \cdot e^{\prime}}{p_{+}}=-2$ becomes free of non-localities.

Although we have demonstrated multiplicative renormalizability of Wilson operators to order $g^{4}$ in the M-L gauge, the complexity of the actual calculation raises the question of usefulness of both, lightcone gauge and Wilson operators as fundamental variables in perturbative QCD. Lightcone gauge has additional problems for loops containing spacelike and/or timelike lines as explained in Appendix C.

\section{Acknowledgment}

A.A. wishes to thank Prof. J.C. Taylor for the invaluable help and advice which made this work possible. The author is grateful to The Royal Society for financial help and D.A.M.T.P. for hospitality. This work was supported by the Ministry of Science and Technology of the Republic of Croatia under Contract No. 00980103.

\section{Appendix A}

There are no transverse components in the Wilson operator as we have assumed in (4). Let us take one of the characteristic integrals which appears in the graph with one 3-gluon vertex in Fig.7.

$$
\begin{gathered}
Z_{\beta}=\int d^{n} k \frac{2 K_{\beta}}{k^{2}(p-k)^{2} k_{+}}\left(e^{-i T(p-k)_{+}}-e^{-i T k_{+}}\right) \\
\times \frac{1}{(p-k)_{-}}\left(e^{-i k_{-} L}-1\right)\left(1-e^{-i(p-k)_{-} L}\right)=P_{\beta} \times M
\end{gathered}
$$

We multiply both sides by the perpendicular momentum $P_{\beta}$, and write

$$
\begin{aligned}
2 P \cdot K= & K^{2}-k_{+} k_{-}-(P-K)^{2}+(p-k)_{+}(p-k)_{-}+p_{+} k_{-}+p_{-} k_{+} \\
Z & \cdot P=P^{2} \times M=\int d^{n} k \frac{(p-k)^{2}-k^{2}+p_{-} k_{+}+p_{+} k_{-}}{k^{2}(p-k)^{2} k_{+}} \\
& \times\left\{e^{-i T p_{+}}\left(e^{i T k_{+}}-1\right)-\left(e^{-i T k_{+}}-1\right)+\left(e^{-i T p_{+}}-1\right)\right\} \\
& \times \frac{1}{(p-k)_{-}}\left\{e^{-i p_{-} L}\left(e^{i(p-k)_{-} L}-1\right)+e^{-i(p-k)_{-} L}-1\right\}
\end{aligned}
$$

In this form we can integrate each of the terms in (A3). $k_{+} p_{-}$gives UV finite term as the integrals of the type

$$
I=\int d^{n} k \frac{1}{k^{2}(p-k)^{2}} e^{i T k_{+}} \frac{1}{(p-k)_{-}}\left(e^{-i(p-k)_{-} L}-1\right)
$$


contain the oscillating factor $e^{i T k_{+}}$which suppresses the possible UV divergences. Let us denote by $Y$ the contribution from $k_{-} p_{+}$.

$$
\begin{gathered}
Y=\int d^{n} k \frac{p_{+} k_{-}}{k^{2}(p-k)^{2} k_{+}} \times\left\{e^{-i T p_{+}}\left(e^{i T k_{+}}-1\right)-\left(e^{-i T k_{+}}-1\right)+\left(e^{-i T p_{+}}-1\right)\right\} \\
\times \frac{1}{(p-k)_{-}}\left\{e^{-i p_{-} L}\left(e^{i(p-k)_{-} L}-1\right)+e^{-i(p-k)_{-} L}-1\right\}
\end{gathered}
$$

The factor $\left(e^{-i T p_{+}}-1\right)$ gives only UV finite term. Also we can write

$$
\frac{k_{-}}{(p-k)_{-}}=\frac{(k-p)_{-}+p_{-}}{(p-k)_{-}}=-1
$$

modulo UV finite terms. We change the variable $p-k=k^{\prime}$ and use the argument analogous to (A4) but now with $k_{+}$and $k_{-}$interchanged. The integral

$$
A=\int d^{n} k \frac{1}{k^{2}(p-k)^{2}} e^{i k_{-} L} \frac{1}{(p-k)_{+}}\left(e^{-i(p-k)_{+} T}-1\right)
$$

is UV finite due to the oscillating factor $e^{i k_{-} L}$.

Therefore the UV divergent part of $Y$ is

$$
\begin{aligned}
& Y=p_{+}\left(e^{-i p_{-} L}+1\right) \int d^{n} k \frac{1}{k^{2}(p-k)^{2}(p-k)_{+}} \\
& \times\left\{e^{-i T p_{+}}\left(e^{i T(p-k)_{+}}-1\right)-\left(e^{-i T(p-k)_{+}}-1\right)\right\} .
\end{aligned}
$$

After the integration over $k_{-}$using the formula

$$
\int d^{n} k \frac{1}{k^{2}(p-k)^{2}}=i \pi^{2-\frac{\epsilon}{2}} \Gamma\left(\frac{\epsilon}{2}\right)\left(-p^{2}-i \eta\right)^{-\frac{\epsilon}{2}} \int_{0}^{1} d x x^{-\frac{\epsilon}{2}}(1-x)^{-\frac{\epsilon}{2}}
$$

where

$$
k_{+}=p_{+} x
$$

we obtain

$$
Y=i \pi^{2-\frac{\epsilon}{2}} \Gamma\left(\frac{\epsilon}{2}\right)\left(-p^{2}-i \eta\right)^{-\frac{\epsilon}{2}}\left(e^{-i p_{-} L}+1\right) \times\left[\left(e^{-i T p_{+}}-1\right) \operatorname{Ci}\left(p_{+} T\right)+i\left(e^{-i T p_{+}}+1\right) \operatorname{Si}\left(p_{+} T\right)\right] .
$$

The remaining two integrals in (A3) we denote by $E$ and $F$.

$$
E=\int d^{n} k \frac{1}{k^{2} k_{+}}\left\{e^{-i T p_{+}}\left(e^{i T k_{+}}-1\right)-\left(e^{-i T k_{+}}-1\right)+\left(e^{-i T p_{+}}-1\right)\right\}
$$




$$
\times \frac{1}{(p-k)_{-}}\left\{e^{-i(p-k)_{-} L}-1+e^{-i p_{-} L}\left(e^{i(p-k)_{-} L}-1\right)\right\} .
$$

The term $\left(e^{-i T p_{+}}-1\right)$ gives vanishing contribution upon the integration in the complex $k_{+}$plane as

$$
\int d^{n} k \frac{1}{k^{2} k_{+}} f\left(p_{-}, k_{-}\right)=0
$$

because both poles lie in the same half-plane with $k_{+}$regulated in the sense of Mandelstam [7]. Other terms have no pole at $k_{+}=0$. For the first $\left(e^{i T k_{+}}-1\right)$ we close the contour in the upper half-plane and pick up a pole at $k_{+}=\frac{K^{2}-i \eta}{k_{-}} \theta\left(-k_{-}\right)$, while for the second $\left(e^{-i T k_{+}}-1\right)$ we close the contour in the lower half-plane and pick up a pole at $k_{+}=\frac{K^{2}-i \eta}{k_{-}} \theta\left(k_{-}\right)$.

$$
\begin{aligned}
E= & i \pi e^{-i T p_{+}} \int_{-\infty}^{0} d k_{-} \int d^{2-\epsilon} K \frac{1}{K^{2}-i \eta}\left(e^{i T \frac{K^{2}-i \eta}{k_{-}}}-1\right) \\
\times & \frac{1}{(p-k)_{-}}\left\{e^{-i(p-k)_{-} L}-1+e^{-i p_{-} L}\left(e^{i(p-k)_{-} L}-1\right)\right\} \\
& -i \pi \int_{0}^{\infty} d k_{-} \int d^{2-\epsilon} K \frac{1}{K^{2}-i \eta}\left(1-e^{-i T \frac{K^{2}-i \eta}{k}}\right) \\
& \times \frac{1}{(p-k)_{-}}\left\{e^{-i(p-k)_{-} L}-1+e^{-i p_{-} L}\left(e^{i(p-k)_{-} L}-1\right)\right\}
\end{aligned}
$$

In the case of the lightlike Wilson loop we can omit the tadpoles in $K^{2}$ of the form

$$
\int d^{2-\epsilon} K \frac{1}{K^{2}-i \eta} f\left(k_{-}, p_{-}\right)=0
$$

This step is not permitted for the spacelike or timelike lines (we explain why in Appendix C). Using the integral

$$
T=\int d^{2-\epsilon} K \frac{1}{K^{2}-i \eta} e^{-i T \frac{K^{2}-i \eta}{k_{-}}}=-\frac{2}{\epsilon} \pi^{1-\frac{\epsilon}{2}}\left(\frac{T}{k_{-}}\right)^{\frac{\epsilon}{2}} e^{\frac{i \pi \epsilon}{4}}
$$

and evaluating the remaining $k_{-}$integrals, we obtain

$$
\begin{gathered}
E=\frac{2}{\epsilon} i \pi^{2-\frac{\epsilon}{2}} e^{\frac{i \pi \epsilon}{4}} T^{\frac{\epsilon}{2}} p_{-}^{-\frac{\epsilon}{2}} \times\left\{\frac{2}{\epsilon}\left(e^{-i T p_{+}}-1\right)\left(e^{-i p_{-} L}+1\right)\right. \\
\left.+\left(e^{-i T p_{+}}-1\right)\left[\left(e^{-i p_{-} L}+1\right) \operatorname{ci}\left(p_{-} L\right)+i\left(e^{-i p_{-} L}-1\right) \operatorname{si}\left(p_{-} L\right)\right]-i \pi\left(e^{-i p_{-} L}-1\right)\right\}
\end{gathered}
$$

The last integral is

$$
F=\int d^{n} k \frac{1}{(p-k)^{2} k_{+}}\left(e^{-i T k_{+}}-e^{-i T(p-k)_{+}}\right) \times \frac{1}{(p-k)_{-}}\left(e^{-i k_{-} L}-1\right)\left(1-e^{-i(p-k)_{-} L}\right) .
$$


Using the same methods as for the integral $E$, but here the auxiliary formula is

$$
\begin{gathered}
\int d^{2-\epsilon} K \frac{1}{p_{+}+\frac{K^{2}-i \eta}{k_{-}}} e^{-i T\left(p_{+}+\frac{K^{2}-i \eta}{k_{-}}\right)} \\
=-\pi^{1-\frac{\epsilon}{2}} k_{-}{ }^{1-\frac{\epsilon}{2}} \operatorname{Ei}\left(-i T p_{+}\right)=-\pi^{1-\frac{\epsilon}{2}} k_{-}{ }^{1-\frac{\epsilon}{2}}\left[\operatorname{ci}\left(p_{+} T\right)-i \operatorname{si}\left(p_{+} T\right)\right]
\end{gathered}
$$

we get

$$
\begin{gathered}
F=-i \pi^{2-\frac{\epsilon}{2}} \Gamma\left(\frac{\epsilon}{2}\right)\left(-p_{+} p_{-}-i \eta\right)^{-\frac{\epsilon}{2}}\left(e^{-i T p_{+}}-1\right) \\
\times\left\{\left(e^{-i p_{-} L}+1\right) \mathrm{Ci}\left(p_{-} L\right)+i\left(e^{-i p_{-} L}-1\right) \operatorname{Si}\left(p_{-} L\right)\right\} \\
+i \pi^{2-\frac{\epsilon}{2}} \Gamma\left(-\frac{\epsilon}{2}\right) L^{\frac{\epsilon}{2}}\left(e^{-i p_{-} L}+1\right) \times\left\{\left(e^{-i T p_{+}}-1\right)\left[\operatorname{ci}\left(p_{+} T\right)+\frac{2}{\epsilon} p_{+}^{-\frac{\epsilon}{2}}-\gamma\right]\right. \\
\left.+i\left(e^{-i T p_{+}}+1\right) \operatorname{si}\left(p_{+} T\right)+i \pi e^{-i T p_{+}}\right\} \\
+i \pi^{2-\frac{\epsilon}{2}} L^{\frac{\epsilon}{2}} p_{+}{ }^{-\frac{\epsilon}{2}}\left(e^{-i T p_{+}}+1\right)\left(e^{-i p_{-} L}-1\right) \times \frac{i \pi}{\epsilon} .
\end{gathered}
$$

The sum of the pole parts in $Z \cdot P$ is

$$
Z \cdot P=Y+(E+F)=0,
$$

hence there are no UV divergences in the transverse momentum $P_{\beta}$. 


\section{Appendix B}

As an example of the complications caused by using the M-L gauge, let us take the diagram shown in Fig.19 which in the Feynman gauge contains no ultra-violet divergences. In the M-L gauge the UV divergent part of this graph is

$$
\begin{aligned}
& G_{\beta \rho}=-2 g^{4} C_{G} \operatorname{Tr}\left(t_{b} t_{d}\right) n_{\beta} n_{\rho} \pi^{2-\frac{\epsilon}{2}}(2 \pi)^{-n} \times\left\{\frac{8}{\epsilon^{2}} \frac{1}{q_{+} p_{+}} e^{-i T q_{+}}\left(e^{-i T p_{+}}-1\right)\left(e^{-i r_{-} L}+1\right)\right. \\
& -\frac{8}{\epsilon^{2}} \frac{1}{q_{+} r_{+}}\left(e^{-i T r_{+}}-1\right)\left(e^{-i r_{-} L}+1\right) \\
& +\frac{2}{\epsilon} \frac{1}{q_{+} p_{+}} e^{-i T q_{+}}\left(e^{-i T p_{+}}-1\right)\left[\left(e^{-i r_{-} L}+1\right)\left(\operatorname{Ci}\left(r_{-} L\right)+2 \ln \left(T L \mu^{2}\right)+i \pi+2 \gamma\right)\right. \\
& \left.+i\left(e^{-i r_{-} L}-1\right) \operatorname{Si}\left(r_{-} L\right)\right] \\
& -\frac{2}{\epsilon} \frac{1}{q_{+} r_{+}}\left(e^{-i T r_{+}}-1\right)\left[\left(e^{-i r_{-} L}+1\right)\left(\operatorname{Ci}\left(r_{-} L\right)+2 \ln \left(T L \mu^{2}\right)+i \pi+2 \gamma\right)+i\left(e^{-i r_{-} L}-1\right) \operatorname{Si}\left(r_{-} L\right)\right] \\
& +\frac{2}{\epsilon} \frac{1}{q_{+} p_{+}} e^{-i T q_{+}}\left[\left(e^{-i r_{-} L}+1\right)\left(\operatorname{Ci}\left(r_{-} L\right)-\operatorname{Ci}\left(q_{-} L\right)-\operatorname{Ci}\left(p_{-} L\right)\right)\right. \\
& \left.+i\left(e^{-i r_{-} L}-1\right)\left(\operatorname{Si}\left(r_{-} L\right)-\operatorname{Si}\left(q_{-} L\right)-\operatorname{Si}\left(p_{-} L\right)\right)\right] \\
& \left.-\frac{4}{\epsilon} \frac{1}{r_{+}^{2}}\left(e^{-i r_{-} L}+1\right)\left[\left(e^{-i T r_{+}}+1\right) \operatorname{Ci}\left(r_{-} L\right)+i\left(e^{-i T r_{+}}-1\right) \operatorname{Si}\left(r_{-} L\right)\right]\right\} \\
& -\frac{8}{\epsilon} \frac{1}{r_{+} r_{-}} g_{\rho \beta} g^{4} C_{G} \operatorname{Tr}\left(t_{b} t_{d}\right) \pi^{2-\frac{\epsilon}{2}}(2 \pi)^{-n} \times\left\{( e ^ { - i T r _ { + } } + 1 ) \left[\left(e^{-i r_{-} L}+1\right) \mathrm{Ci}\left(r_{-} L\right)\right.\right. \\
& \left.\left.+i\left(e^{-i r_{-} L}-1\right)\left(\operatorname{Si}\left(r_{-} L\right)-\pi\right)\right]+2 i \pi\left(e^{-i r_{-} L}-1\right)\right\} \\
& +\frac{4}{\epsilon} g^{4} C_{G} \operatorname{Tr}\left(t_{b} t_{d}\right) n_{\beta}^{*} n_{\rho} \pi^{2-\frac{\epsilon}{2}}(2 \pi)^{-n} \frac{1}{q_{+} r_{-}} \times\left\{( e ^ { - i T q _ { + } } - 1 ) \left[\left(e^{-i r_{-} L}+1\right)\left(\operatorname{Ci}\left(p_{-} L\right)-\operatorname{Ci}\left(q_{-} L\right)\right)\right.\right. \\
& \left.+i\left(e^{-i r_{-} L}-1\right)\left(\operatorname{Si}\left(p_{-} L\right)-\operatorname{Si}\left(q_{-} L\right)+\pi\right)\right] \\
& \left.-\left(e^{-i r_{-} L}+1\right)\left[\operatorname{Ci}\left(r_{-} L\right)-\operatorname{Ci}\left(p_{-} L\right)+\operatorname{Ci}\left(q_{-} L\right)\right]-i\left(e^{-i r_{-} L}-1\right)\left[\operatorname{Si}\left(r_{-} L\right)-\operatorname{Si}\left(p_{-} L\right)+\operatorname{Si}\left(q_{-} L\right)\right]\right\} \\
& -\frac{4}{\epsilon} g^{4} C_{G} \operatorname{Tr}\left(t_{b} t_{d}\right) n_{\rho}^{*} n_{\beta} \pi^{2-\frac{\epsilon}{2}}(2 \pi)^{-n} \frac{1}{p_{+} r_{-}} e^{-i T q_{+}} \\
& \times\left\{\left(e^{-i T p_{+}}-1\right)\left[\left(e^{-i r_{-} L}+1\right) \mathrm{Ci}\left(r_{-} L\right)+i\left(e^{-i r_{-} L}-1\right)\left(\operatorname{Si}\left(r_{-} L\right)-\pi\right)\right]\right. \\
& \left.+\left(e^{-i r_{-} L}+1\right)\left[\mathrm{Ci}\left(p_{-} L\right)-\operatorname{Ci}\left(q_{-} L\right)+\operatorname{Ci}\left(r_{-} L\right)\right]+i\left(e^{-i r_{-} L}-1\right)\left[\operatorname{Si}\left(p_{-} L\right)-\operatorname{Si}\left(q_{-} L\right)+\operatorname{Si}\left(r_{-} L\right)\right]\right\}
\end{aligned}
$$




\section{Appendix $\mathrm{C}$}

In the case of Wilson loops with spacelike and/or timelike lines strict application of dimensional regularization is not possible. As an example let us take the self-energy type of graph in the triangle Wilson loop with one spacelike* and two lightlike sides shown in Fig.20.

$$
\begin{gathered}
W_{\beta \rho}=C_{\beta \rho} \int d^{n} k \frac{1}{k^{2}+i \eta} \frac{k_{-}}{k_{+}+i \omega k_{-}} \frac{1}{k_{3} p_{3}} \\
\times\left\{\frac{1}{(p-k)_{3}} e^{-i p_{3} L}\left(e^{i(p-k)_{3} L}-1\right)+\frac{1}{(p+k)_{3}}\left(e^{-i(p+k)_{3} L}-1\right)\right\} \\
=C_{\beta \rho} W
\end{gathered}
$$

where

$$
C_{\beta \rho}=-i g^{4} v_{\beta} n_{\rho} \operatorname{Tr}\left(t_{b} t_{d}\right)(2 \pi)^{-n} \frac{1}{q_{+}}\left(e^{\frac{i q_{+} L}{2}}-1\right) e^{-\frac{i q_{-} L}{2}}
$$

There are two poles in the upper-half complex $k_{0}$ plane.

(a)

$$
\begin{gathered}
k^{2}+i \eta=0, \\
k_{0}=-k+i \eta
\end{gathered}
$$

(b)

$$
\begin{gathered}
k_{+}+i \omega k_{-}=0, \\
k_{0}=-k_{3}+2 i \omega k_{3} \theta\left(k_{3}\right)
\end{gathered}
$$

Let us take the first part of $W$ with the $\frac{1}{(p-k)_{3}}$ denominator. After the $k_{0}$ integration it gives

$$
\begin{aligned}
& W_{1}=2 i \pi e^{-i p_{3} L} \int d k_{3} d^{2-\epsilon} K \frac{1}{2 k} \frac{k+k_{3}}{k-k_{3}+i \omega\left(k+k_{3}\right)} \\
& \times \frac{1}{k_{3} p_{3}(p-k)_{3}}\left\{\cos (p-k)_{3} L-1+i \sin (p-k)_{3} L\right\} \\
& \quad+2 i \pi e^{-i p_{3} L} \int_{0}^{\infty} d k_{3} \int d^{2-\epsilon} K \frac{2 k_{3}}{K^{2}+4 i \omega k_{3}^{2}-i \eta} \\
& \times \frac{1}{k_{3} p_{3}(p-k)_{3}}\left\{\cos (p-k)_{3} L-1+i \sin (p-k)_{3} L\right\}
\end{aligned}
$$

* This feature of the M-L prescription was noticed already in A. Andraši, hep-th 9411117, unpublished 
Naively, one would strictly apply the rules of dimensional regularization and set the second integral to zero as a tadpole in the perpendicular momentum $K$. However, after the introduction of polar coordinates

$$
\begin{gathered}
k_{3}=k \cos \theta=k x \\
d^{3-\epsilon} k=k^{2-\epsilon} d k\left(1-x^{2}\right)^{-\frac{\epsilon}{2}} d x \int d \Phi \\
\int d \Phi=\frac{2 \pi^{1-\frac{\epsilon}{2}}}{\Gamma\left(1-\frac{\epsilon}{2}\right)}
\end{gathered}
$$

and integration over $k$, the first integral leads to an integral which is not defined for any $\epsilon$.

Therefore we have to keep $\omega$ in the integrand and it becomes a part of the gauge. We can choose two ways. Either we evaluate integrals separately in terms of the spurious, 'ambiguous' terms of the form $\omega^{-\frac{\epsilon}{2}} \epsilon^{-2}$ dictated by the tadpole

$$
W^{T}=\int d^{2-\epsilon} K \frac{1}{K^{2}+4 i \omega k_{3}^{2}-i \eta}=\pi^{1-\frac{\epsilon}{2}} \Gamma\left(\frac{\epsilon}{2}\right) \omega^{-\frac{\epsilon}{2}} 2^{-\epsilon} e^{\frac{-i \pi \epsilon}{4}} k_{3}^{-\epsilon}
$$

or we transform the tadpole into polar coordinates

$$
W^{T}=\int_{0}^{\infty} d k k^{-\epsilon} \int_{0}^{1} d x\left(1-x^{2}\right)^{-\frac{\epsilon}{2}} \frac{1}{1-x^{2}+4 i \omega x^{2}} \int d \Phi
$$

and sum it up with the first integral in (C4) leading to

$$
\begin{gathered}
W_{1}=-i \pi e^{-i p_{3} L} p_{3}^{-1-\epsilon}\left[\operatorname{ci}\left(p_{3} L\right)+\frac{1}{\epsilon}+i \operatorname{si}\left(p_{3} L\right)+i \pi\right] \int d \Phi \\
\times \int_{0}^{1} d x\left(1-x^{2}\right)^{-\frac{\epsilon}{2}} x^{\epsilon-2}\left[\frac{2\left(1+x^{2}\right)}{1-x^{2}+2 i \omega\left(1+x^{2}\right)}-\frac{4 x}{1-x^{2}+4 i \omega x^{2}}\right] \\
+(i \pi)^{2} e^{-i p_{3} L} p_{3}^{-1-\epsilon} \int_{0}^{1} d x\left(1-x^{2}\right)^{-\frac{\epsilon}{2}} x^{\epsilon-2} \frac{1-x}{1+x+i \omega(1-x)} \int d \Phi .
\end{gathered}
$$

We notice how crucial the contribution from the tadpole $-\frac{4 x}{1-x^{2}+4 i \omega x^{2}}$ is for the regularization of the pole at $x=1$. Only after the addition of the tadpole we can set $\omega=0$ in (C8) and evaluate the integrals in the strip $1<\epsilon<4$. In the same way we evaluate the second part of $(\mathrm{C} 1)$ with $\frac{1}{(p+k)_{3}}$ denominator.* $^{*}$

* Let us mention that the change of variable $k_{3}$ into $-k_{3}$ in the second part of $(\mathrm{C} 1)$ is not permitted as it creates the pole in $k_{3}$. 
Thus we obtain the result for $(\mathrm{C} 1)$

$$
\begin{gathered}
W_{\beta \rho}=-C_{\beta \rho} \frac{4 i \pi^{2-\frac{\epsilon}{2}}}{\Gamma\left(1-\frac{\epsilon}{2}\right)} p_{3}^{-1-\epsilon} 2^{-\epsilon}\left[\frac{2}{\epsilon}+1\right] \\
\times\left\{\left(e^{-i p_{3} L}-1\right)\left(\operatorname{ci}\left(p_{3} L\right)+\frac{1}{\epsilon}\right)+i\left(e^{-i p_{3} L}+1\right)\left(\operatorname{si}\left(p_{3} L\right)+\frac{\pi}{2}\right)\right\} .
\end{gathered}
$$

This graph in the Feynman gauge contains only simple single poles. Hence, the funny non-local sine and cosine divergences and the double pole are caused by the choice of the M-L gauge, not the lightlike sides of the loop.

\section{References}

[1] A. Andraši and J.C. Taylor, Nucl. Phys.B516(1998)417;

[2] V.S. Dotsenko and S.N. Vergeles, Nucl. Phys. B169(1980)527;

[3] R. Brandt, F. Neri and Masa-aki Sato, Phys. Rev.D24(1981)879;

[4] G. Leibbrandt, Phys. Rev. D29(1984)699;

[5] J.G.M. Gatheral, Phys. Lett.133B(1983)90;

[6] J. Frenkel and J.C. Taylor, Nucl. Phys.B246(1984)231;

[7] S. Mandelstam, Nucl. Phys.B213(1983)149;

[8] A. Andraši, G. Leibbrandt and S.L. Nyeo, Nucl. Phys.B276 (1986)445;

[9] A. Andraši and J.C. Taylor, Nucl. Phys.B302(1988)123;

[10] P. Gaigg, W. Kummer and M. Schweda (Eds.),

Physical and Nonstandard Gauges, Proceedings, Vienna, Austria 1989 


\section{Figure Captions}

Fig.1. Wilson operator at order $g^{4}$ with two real gluons and one 3 -gluon vertex. The sides of the loop are along the lightlike vectors used to define the M-L prescription, $n^{*}$ of length $L$ and $n$ of length $T$. The two graphs of the $A$-set which contribute to the $n^{*} n^{*}$ sector have their symmetric counterparts.

Fig.2. $B$-set of graphs.

Fig.3. $C$-set.

Fig.4. $D$-set

Fig.5. E-set

Fig.6. Graphs with two 3-gluon vertices and a graph with the 4-gluon vertex which contribute to the $n n$ sector of the Wilson operator at order $g^{4}$.

Figs.7 and 8. G1-set of graphs. Graphs with one 3-gluon vertex which contribute to the $n n$ sector.

Fig.9. Left and right graphs with two 3-gluon vertices in the $G(L+R)$-set which contribute to the $n n$ sector.

Fig.10. $G 0$-set of graphs in the $n n$ sector.

Fig.11. The $A$-set of graphs which contribute to the $n_{\beta} n_{\rho}^{*}$ sector.

Fig.12. The $A^{\prime}$-set of graphs.

Fig.13. $B$-set of graphs in the $n_{\beta} n_{\rho}^{*}$ sector.

Fig.14. $C$-set of graphs.

Fig.15. $C^{\prime}$-set.

Fig.16. $D$-set.

Fig.17. E-set.

Fig.18. $F$-set of graphs which contribute to the $n_{\beta} n_{\rho}^{*}$ sector.

Fig.19. The graph with two 3 -gluon vertices which in the Feynman gauge contains no UV divergences. The same graph in the $\mathrm{M}-\mathrm{L}$ gauge contains double divergences and single divergences with non-local $\mathrm{Ci}$ and Si functions.

Fig.20. The triangle Wilson operator. The base is along the spacelike vector $v_{\beta}$ of length $L$, while the sides are along the lightlike vectors $n$ and $n^{*}$ of length $\frac{L}{2}$. 


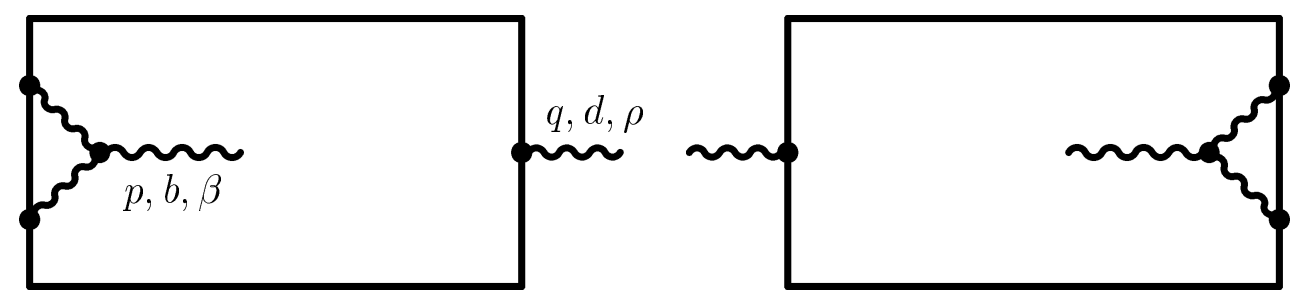

Fig.1

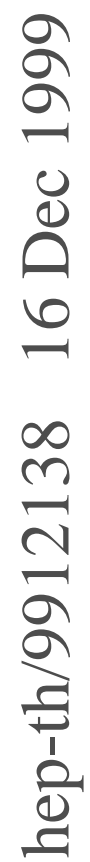
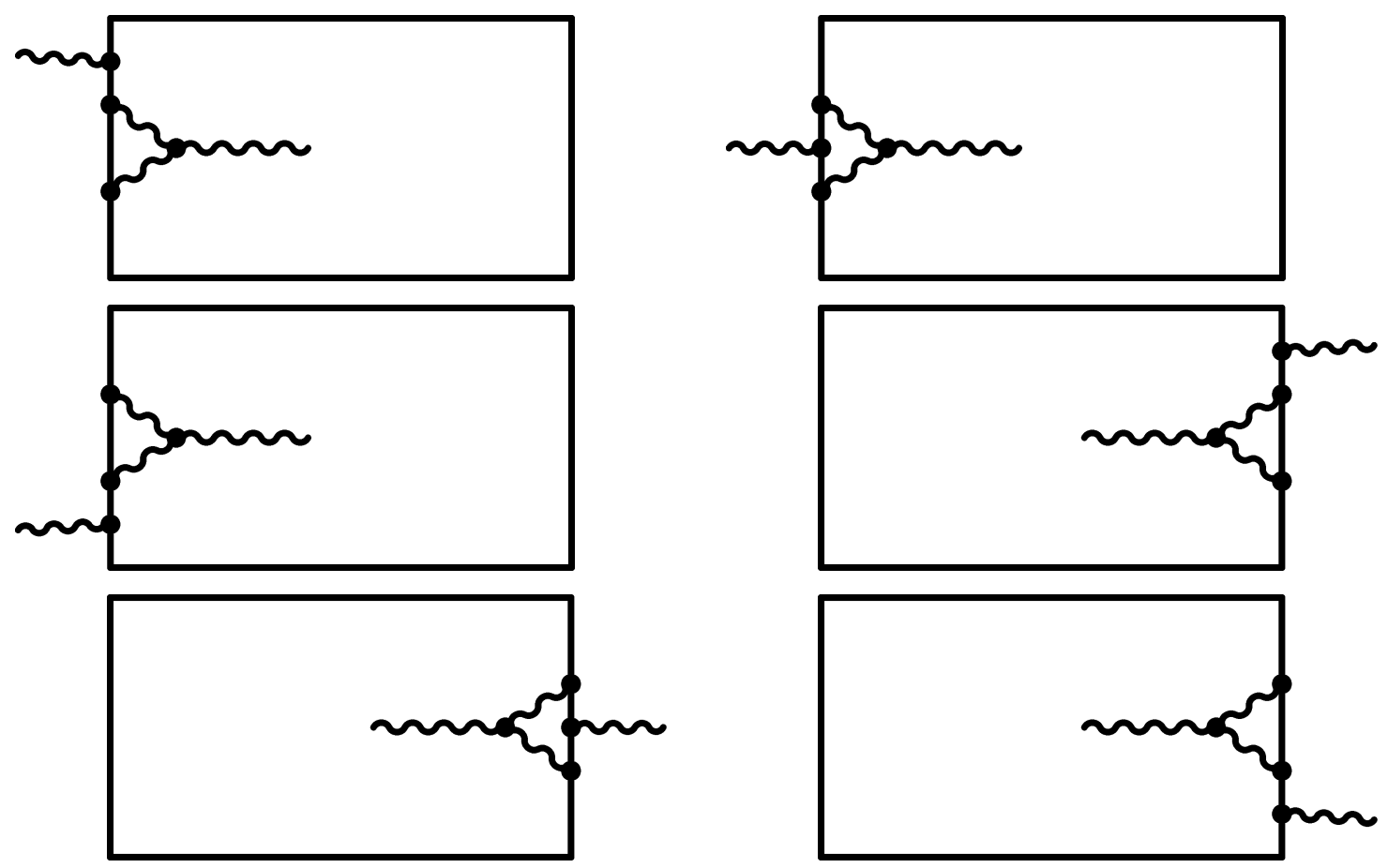

Fig. 2 

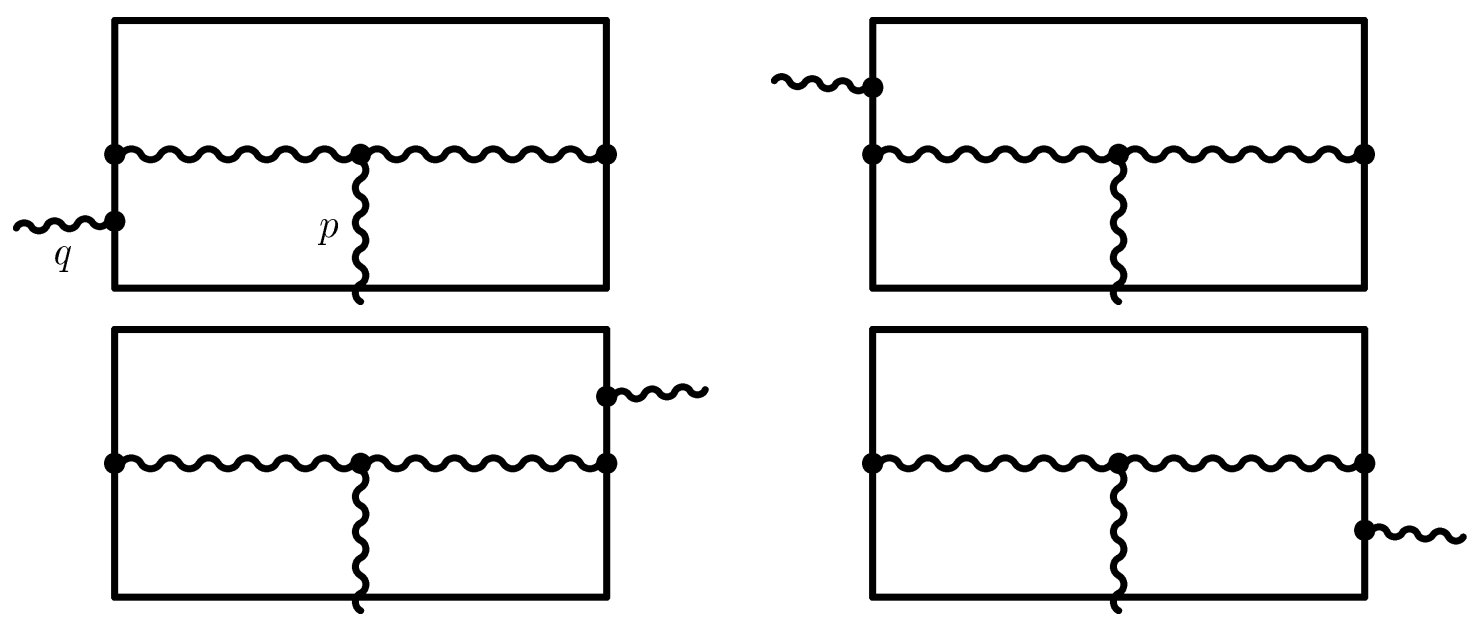

Fig.3
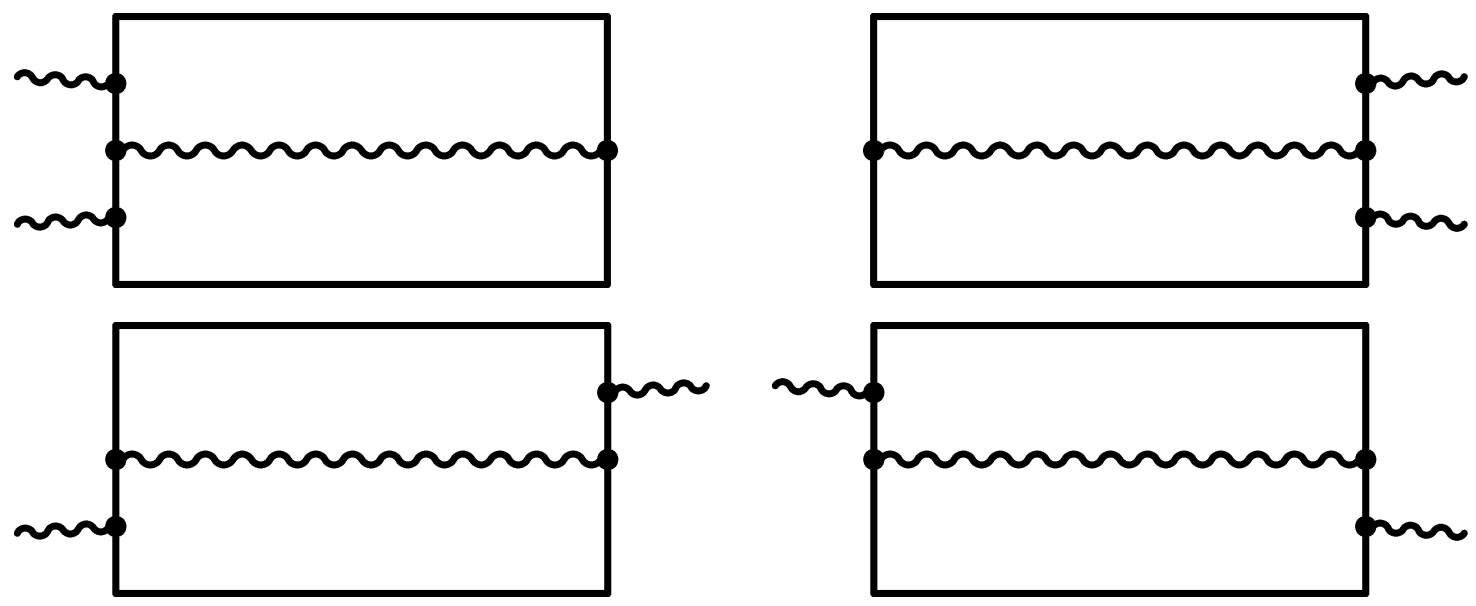

Fig.4 

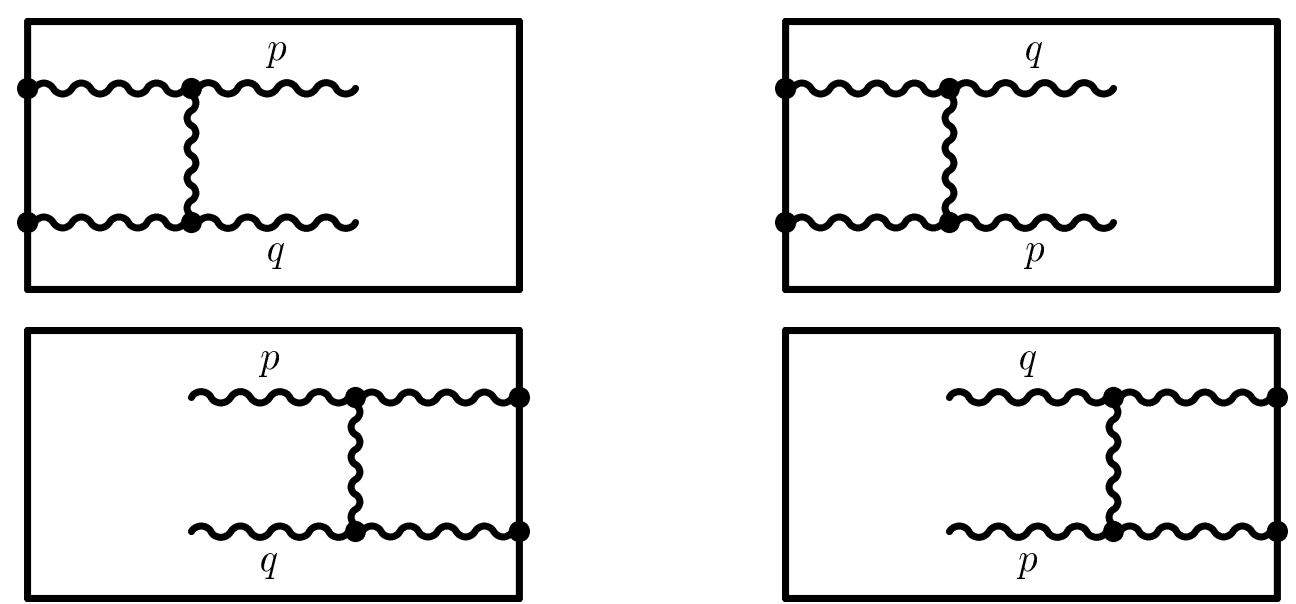

Fig.5
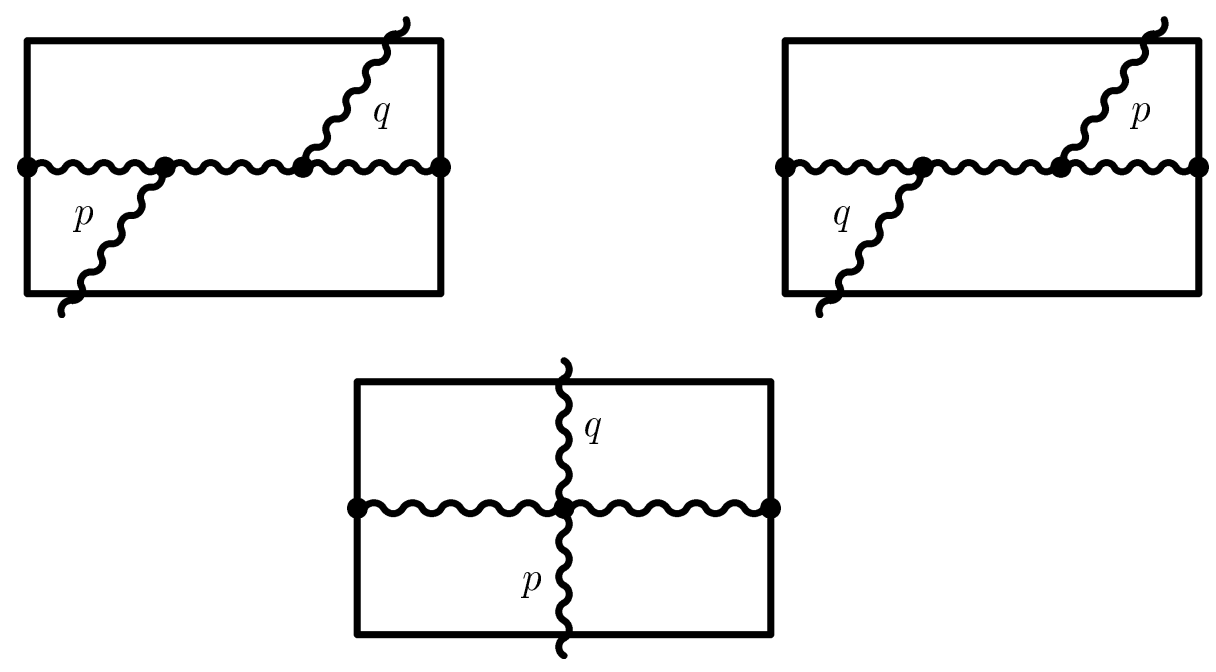

Fig.6 

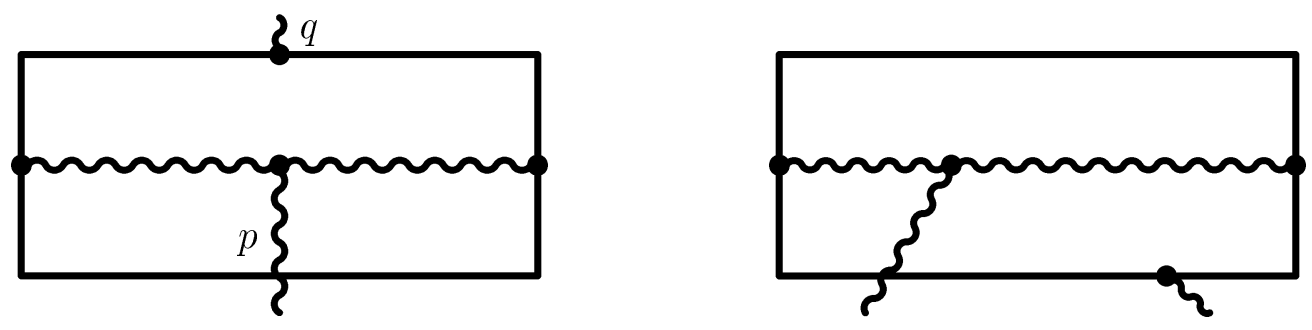

Fig.7
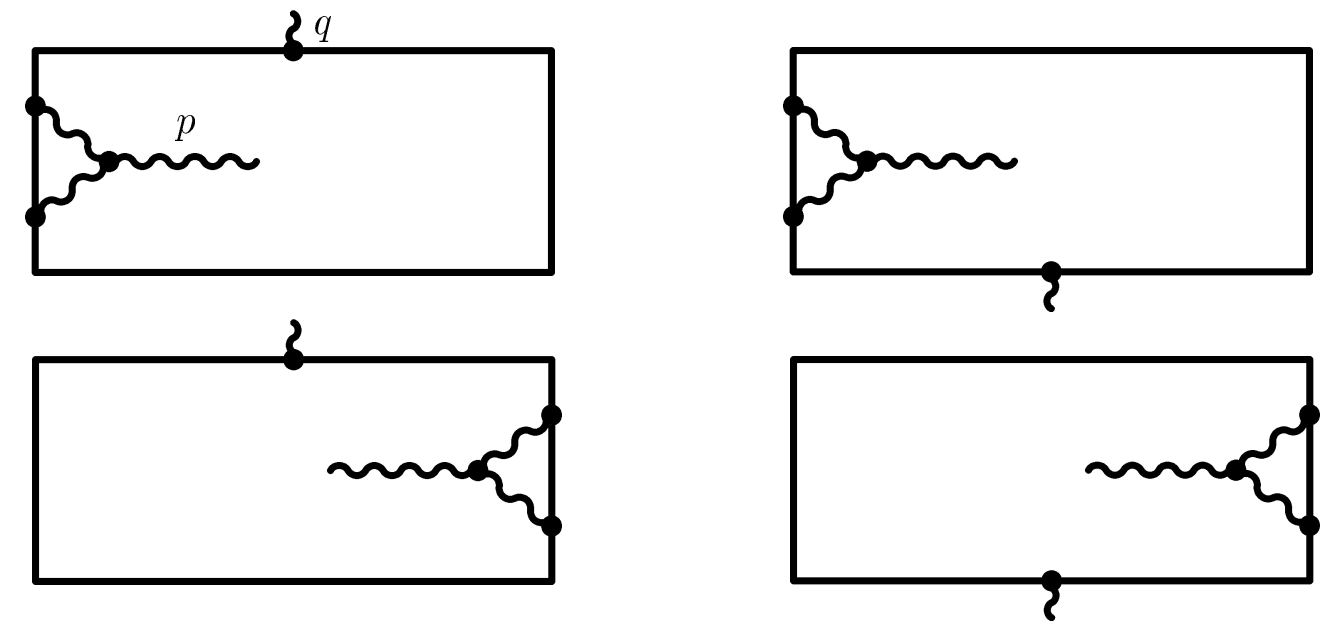

Fig.8 

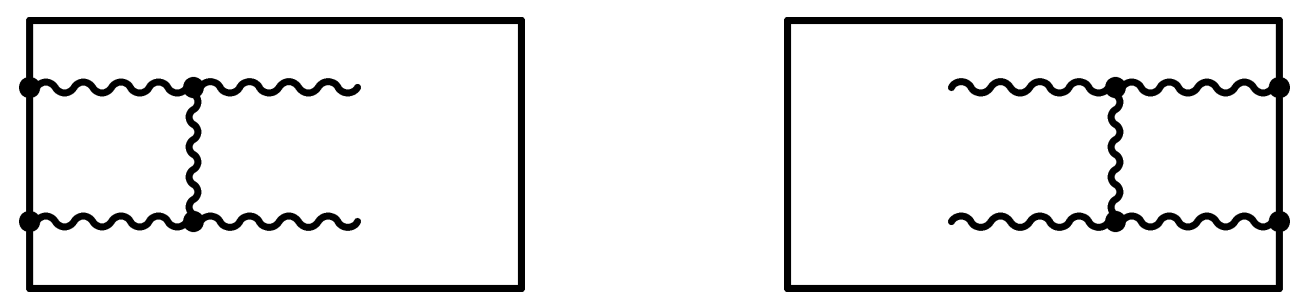

Fig.9
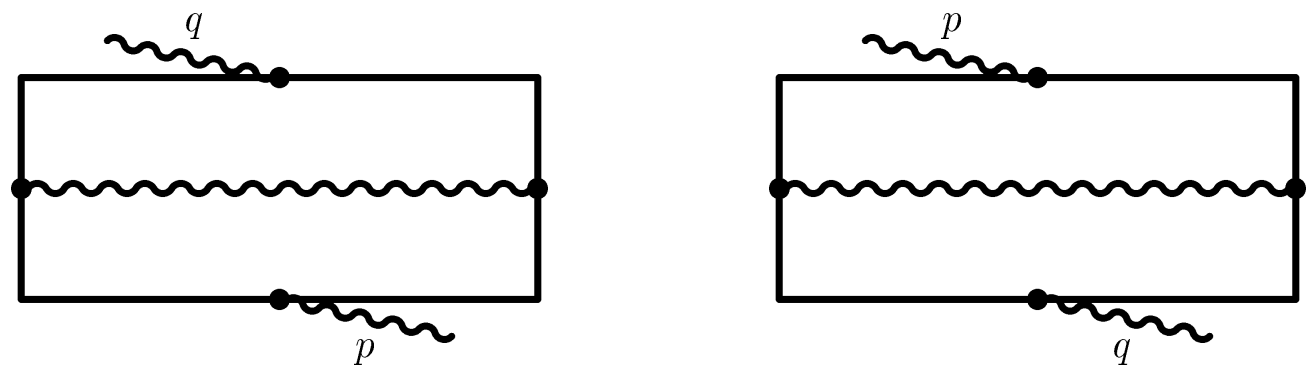

Fig.10

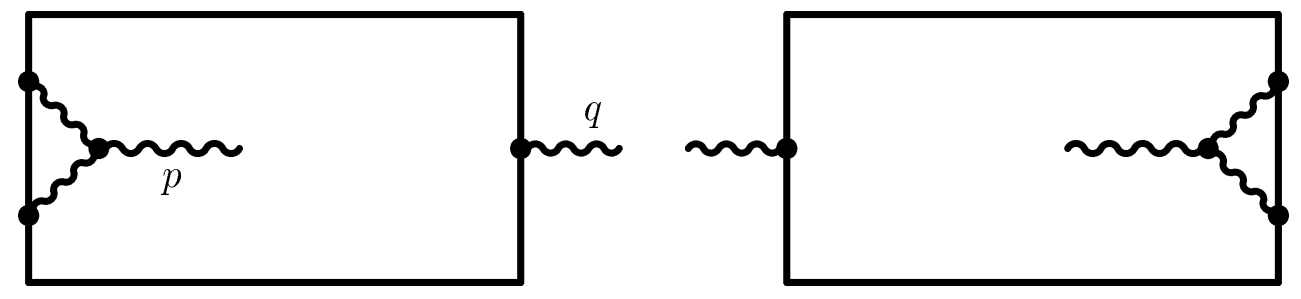

Fig.11 

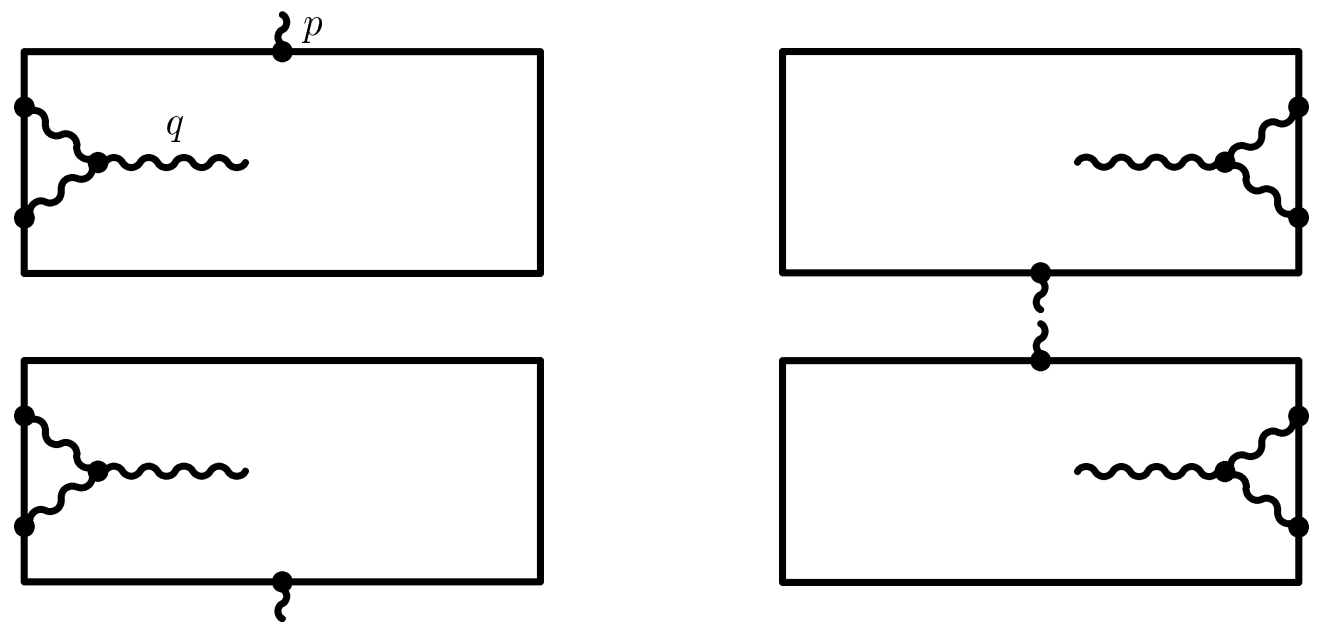

Fig.12 

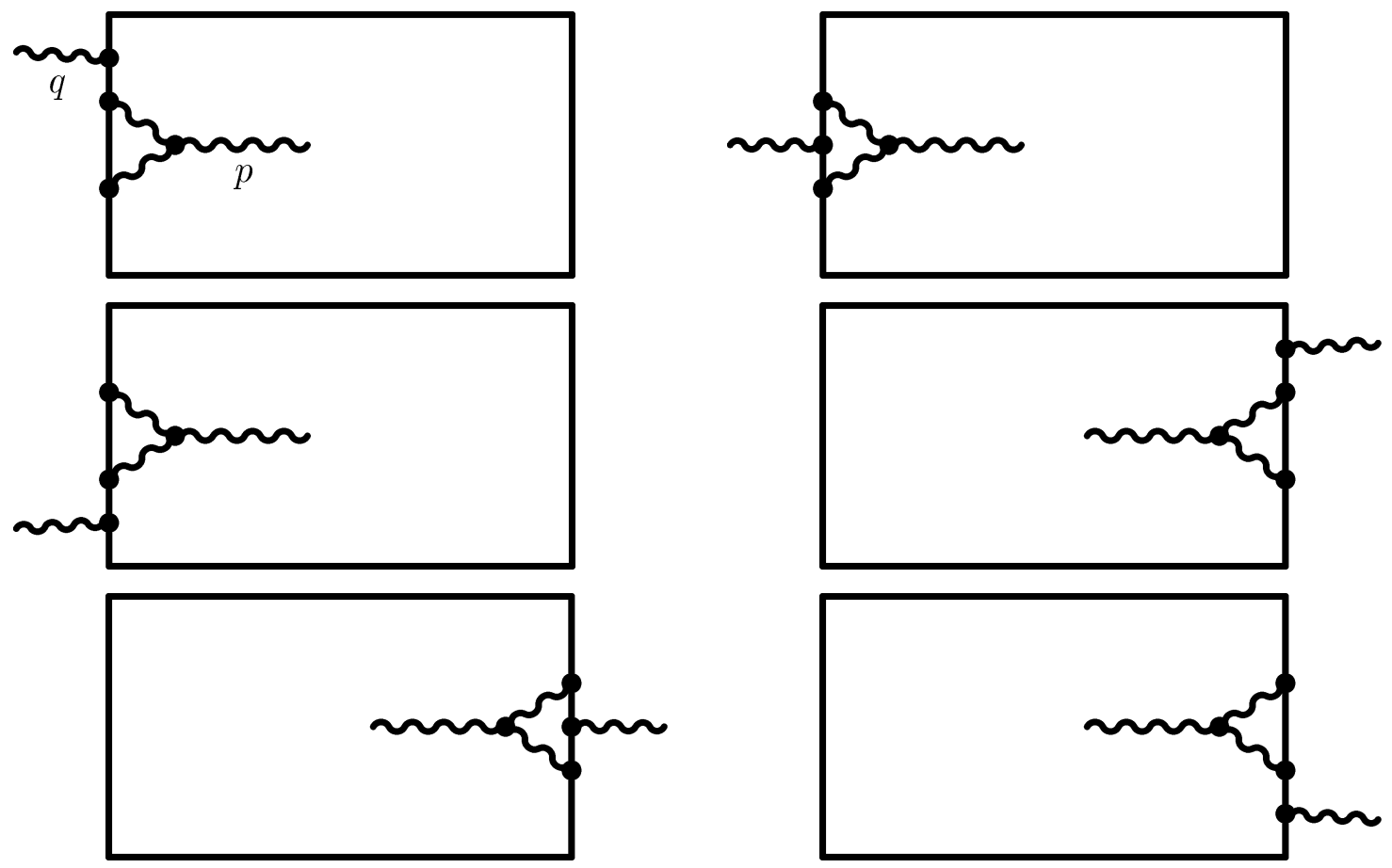

Fig.13 

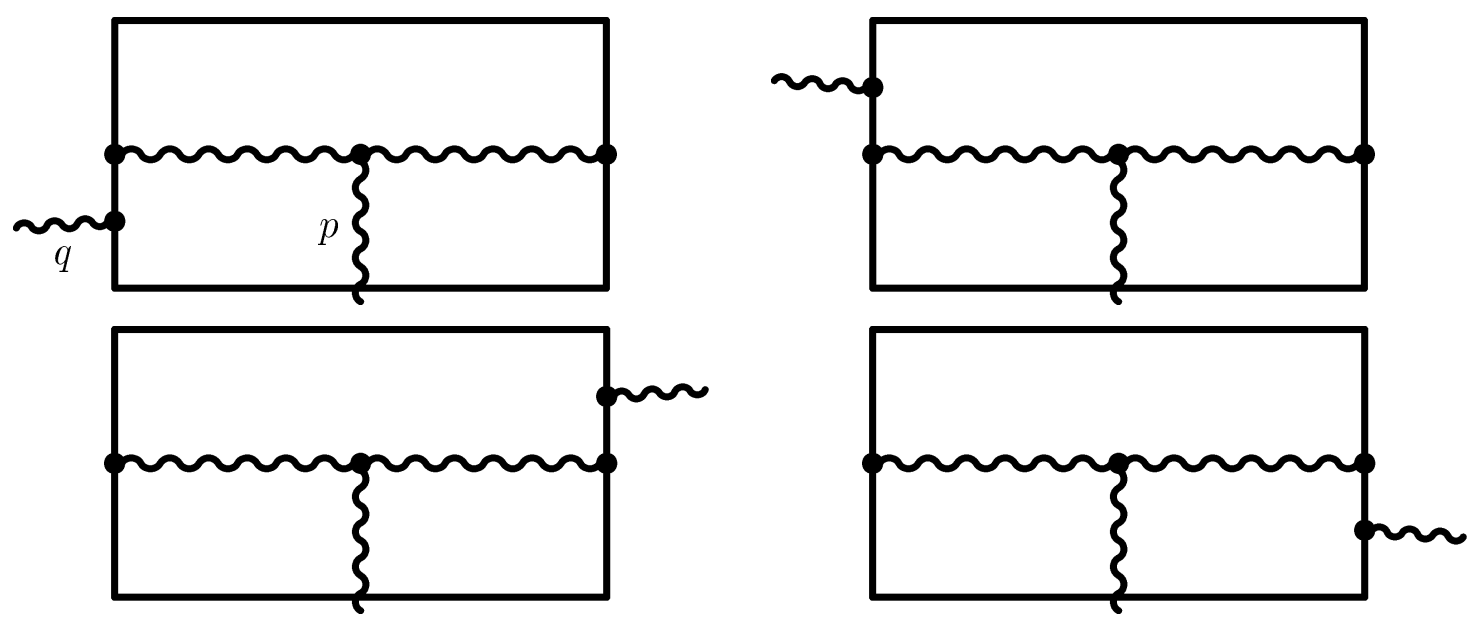

Fig.14 

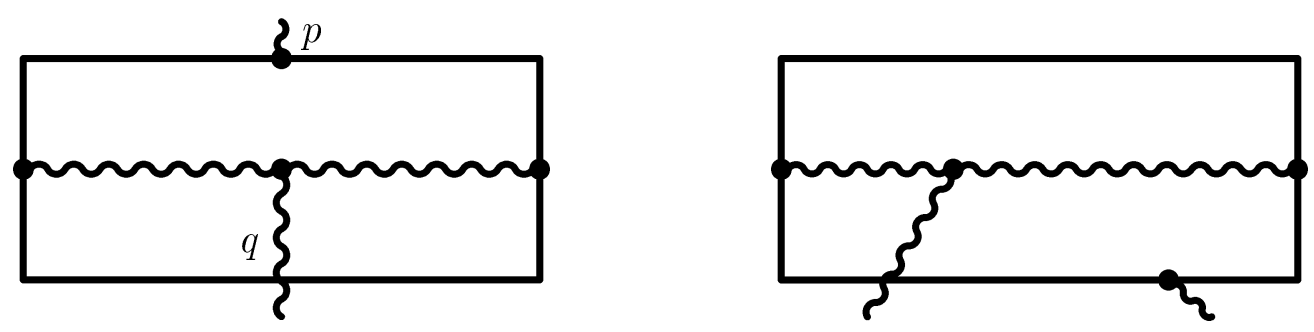

Fig.15
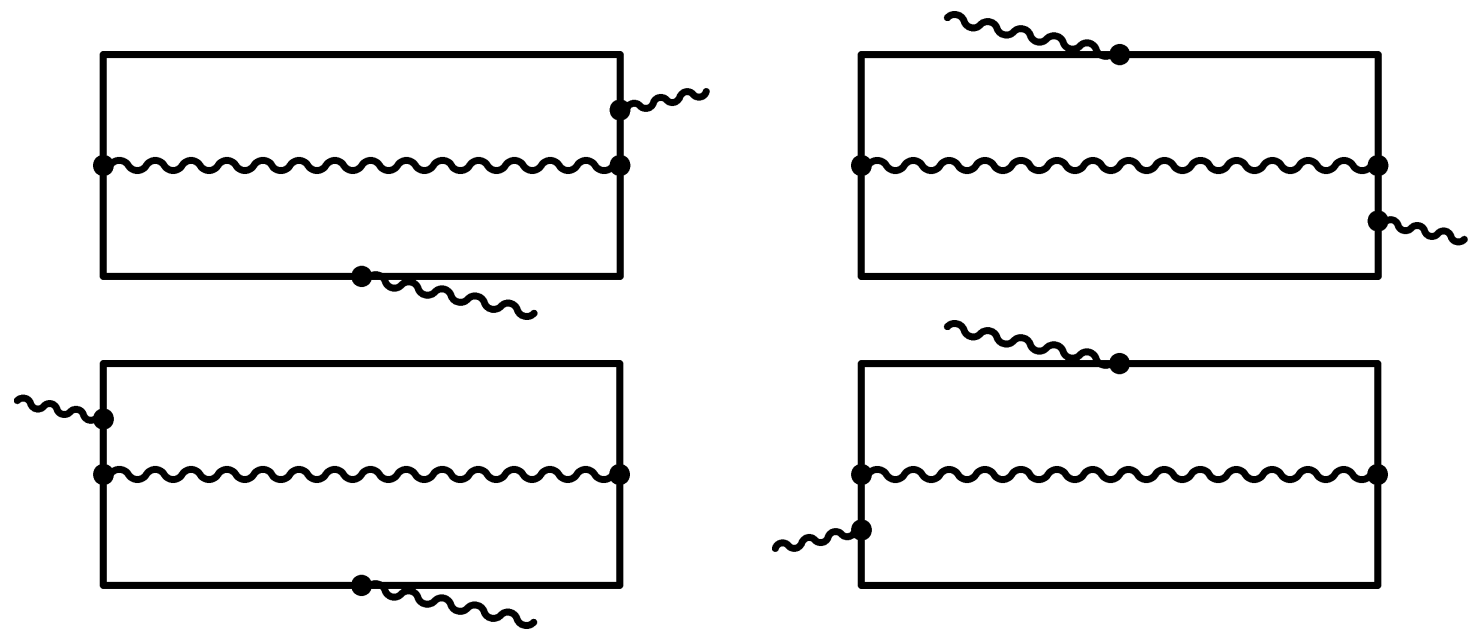

Fig.16 

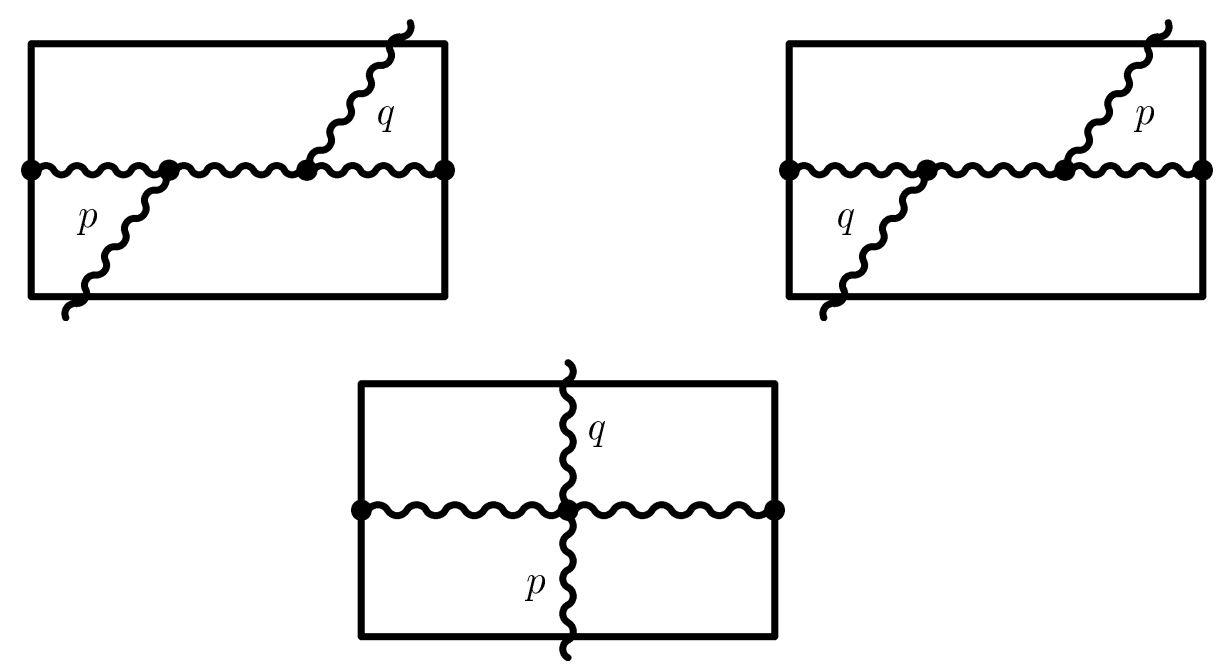

Fig.17
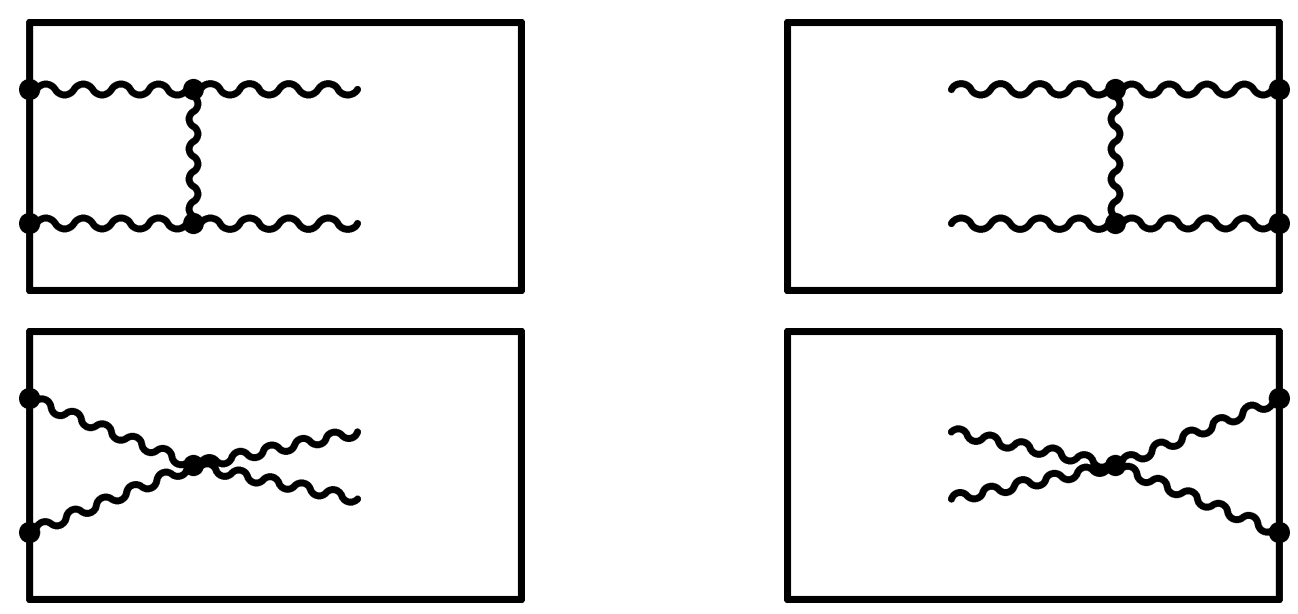

Fig.18 


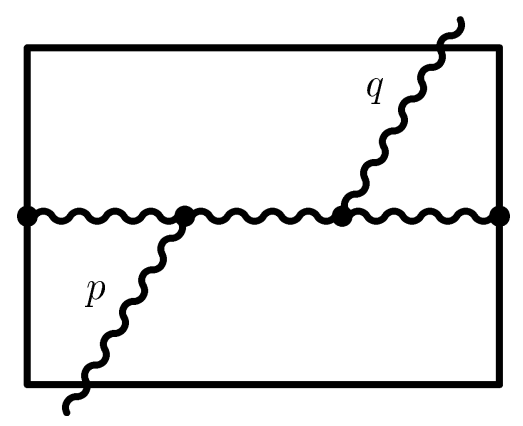

Fig.19

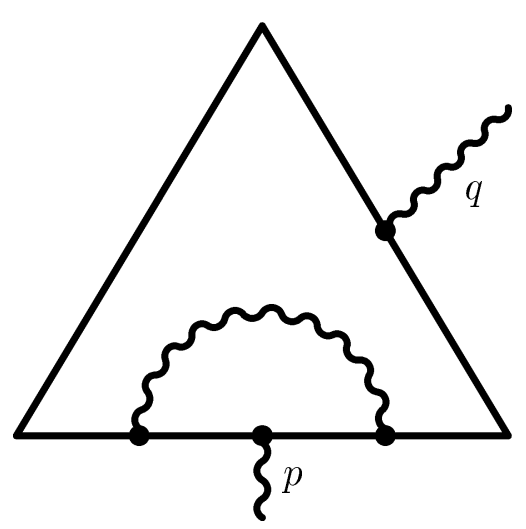

Fig. 20 